\title{
Advances in the understanding and treatment of sepsis-induced immunosuppression
}

\section{Fabienne Venet and Guillaume Monneret}

Abstract $\mid$ Sepsis is defined as a life-threatening organ dysfunction that is caused by a dysregulated host response to infection. Sepsis can induce acute kidney injury and multiple organ failures and represents the most common cause of death in the intensive care unit. Sepsis initiates a complex immune response that varies over time, with the concomitant occurrence of both pro-inflammatory and anti-inflammatory mechanisms. As a result, most patients with sepsis rapidly display signs of profound immunosuppression, which is associated with deleterious consequences. Scientific advances have highlighted the role of metabolic failure, epigenetic reprogramming, myeloid-derived suppressor cells, immature suppressive neutrophils and immune alterations in primary lymphoid organs (the thymus and bone marrow) in sepsis. An improved understanding of the mechanisms underlying this immunosuppression as well as of the similarities between sepsis-induced immunosuppression and immune defects in cancer or immunosenescence has led to novel therapeutic strategies aimed at stimulating immune function in patients with sepsis. Trials assessing the therapeutic benefit of IL-7, granulocytemacrophage colony-stimulating factor (GM-CSF) and antibodies against programmed cell death protein 1 (PD1) and programmed cell death 1 ligand 1 (PDL1) for the treatment of sepsis are in progress. The reappraisal of sepsis pathophysiology has also resulted in a novel approach to the design of clinical trials evaluating sepsis treatments, based on an evaluation of the immune status and biomarker-based stratification of patients.

Hospices Civils de Lyon, Hôpital Edouard Herriot, Immunology Department, Flow Division, 69003 Lyon, France.

Equipe d'Accueil 7426, Pathophysiology of InjuryInduced Immunosuppression, Université Claude Bernard Lyon 1, Hospices Civils de Lyon - bioMérieux, Hôpital Edouard Herriot, 69003 Lyon, France.

Correspondence to G.M. guillaume.monneret@ chu-lyon.fr
Sepsis is defined as a life-threatening organ dysfunction that is caused by a dysregulated host response to infection. Septic shock is a subtype of sepsis in which hypotension persists despite adequate volume resuscitation, and its treatment requires the use of vasopressors ${ }^{1}$. Sepsis represents a major, under-recognized health-care problem worldwide and causes a high number of deaths every year ${ }^{2,3}$. Consequently, sepsis has been described as "the quintessential medical disorder of the 21st century" (REF. 2), and on 26 May 2017, the World Health Organization declared sepsis a global health priority by adopting a resolution to improve the prevention, diagnosis and management of this deadly disease $e^{4}$. The host immune response during and after sepsis is complex and involves an initial excessive host inflammatory response to infection that is associated with tissue damage and leads to organ failure and endothelial dysfunction $^{5}$. Acute kidney injury is a common form of tissue damage in sepsis and is often severe enough to require renal replacement therapy. Nevertheless, decades of trials using anti-inflammatory treatment approaches to control the devastating effects of this excessive inflammation have been unsuccessful, which has led clinicians and researchers to revisit the pathophysiology of sep$s^{6}{ }^{6}$. Although initially not appreciated, the occurrence of severe immunosuppression in patients with sepsis (either concomitantly with the initial inflammation or delayed) is now a well-established phenomenon ${ }^{7,8}$ and constitutes an important area of research that should rapidly offer novel tailored immunotherapies with similarities to those that are being evaluated in the treatment of various cancers. In this Review, we discuss the main characteristics of sepsis-induced immunosuppression, including those at the cellular level, and its clinical consequences. In addition, we consider innovative aspects in the development of therapies that target the host response, including the design and use of preclinical animal models of sepsis and of clinical trials based on biomarker stratification of patients. Finally, we discuss the most promising candidates for immunotherapy (that 


\section{Key points \\ - Sepsis is defined as a life-threatening organ dysfunction that is caused by a dysregulated host response to infection and represents the major cause of death in the intensive care unit \\ - Acute kidney injury is a common organ dysfunction during sepsis, as a consequence of an initial exaggerated inflammatory response \\ - Sepsis induces a marked and long-term immunosuppression that shares similarities with immunosenescence or the immune defects described in cancer \\ - Immune cell metabolism, epigenetic reprogramming, immune checkpoint inhibitors, suppressive myeloid cells and long-term persistence of immune defects are the focus of current research \\ - Immunostimulation is a novel therapeutic option in the most immunosuppressed patients with sepsis; results of clinical trials evaluating IL-7 and granulocyte- macrophage colony-stimulating factor (GM-CSF) in the treatment of sepsis are expected to be published in 2018 \\ - Companion biomarkers are desirable to evaluate immune status, better stratify patients and control immunostimulation effectiveness in a tailored approach to the treatment of sepsis}

in patients who died of sepsis ${ }^{12,13}$. Increased apoptosis of T cells, B cells and dendritic cells leads to a marked reduction in their abundance in the circulation of patients. Both intrinsic (mitochondria-mediated) apoptosis and extrinsic (death-receptor-mediated) apoptosis are activated after sepsis ${ }^{17}$. In addition, apoptotic cells are immunosuppressive and tolerogenic in nature, which affects the maturation and functions of dendritic cells in mice with sepsis ${ }^{18}$. The increased apoptosis could thus contribute to a reorientation of the immune system towards an anti-inflammatory immunosuppressive response. In mice, numerous anti-apoptotic strategies have been successful in decreasing mortality after sepsis $^{19,20}$. In addition, alternative forms of programmed cell death, such as pyroptosis or necroptosis, are induced after sepsis ${ }^{21}$. Although the role of these pathways in the initiation of the inflammatory response and the efficient clearance of infection has been established, their participation in sepsis-induced immune alterations is unknown. Autophagy, which is a catabolic process that involves lysosomal degradation and recycling of cytoplasmic constituents, has also emerged as a novel mediator of cell death $^{22}$ (see below).

\section{Autophagy}

Autophagy can be a cytoprotective process and can also induce cell death. Furthermore, crosstalk can occur between autophagy and apoptosis ${ }^{23}$. In the caecal ligation and puncture (CLP) mouse model of sepsis, apoptosis of $\mathrm{T}$ cells is induced, whereas autophagy is downregulated in $\mathrm{CD}^{+}$splenocytes and $\mathrm{CD}^{+}$splenocytes $^{24,25}$. In addition, mice with lymphocyte-specific deletion of Atg5 or Atg7 - genes that are essential for autophagy - have increased mortality, immune dysfunction and $\mathrm{T}$ cell apoptosis compared with that of control mice ${ }^{24,25}$. For example, T cell-specific deletion of Atg5 in mice results in increased IL-10 production by T cells after CLP ${ }^{25}$. These findings suggest that deficient autophagy contributes to sepsis-induced immunosuppression.

\section{Endotoxin tolerance}

Long-term exposure to LPS or injection of sublethal doses of LPS in animals can induce a state of tolerance that reprogrammes the inflammatory response, resulting in reduced inflammatory cytokine production in vitro and in vivo in response to subsequent challenge with LPS or other inflammatory stimuli ${ }^{26}$. In vitro, many myeloid cells lose their ability to respond to LPS following an initial challenge. This phenomenon has been called LPS or endotoxin tolerance and is a conserved and important biological phenomenon ${ }^{27}$. The key readout for endotoxin tolerance is a reduction in TNF production in tolerized cells compared with that of unprimed cells. Endotoxin-tolerant monocytes also exhibit an increased phagocytic ability coupled with a conserved capacity to kill internalized pathogens, albeit with impaired antigen-presentation and chemotaxis capacities $^{28,29}$. Endotoxin tolerance is characterized by notable changes in transcription, with decreased expression of genes encoding pro-inflammatory cytokines and chemokines (termed 'tolerizable genes'). Evidence also 
suggests that LPS tolerance increases the expression of a subset of genes (termed 'non-tolerizable genes') that may protect animals from systemic infection while they are in an immunosuppressed state $e^{29-31}$. This increased expression results in a shift towards the production of immunosuppressive molecules that act at a systemic level, such as IL-10 (REFS 32,33). Cell-intrinsic and epigenetic mechanisms participate in the transcriptional reprogramming observed in tolerized cells $\mathrm{s}^{27,32,34}$. For example, the glycoprotein $\mathrm{G}$ protein-coupled receptor 84 (GPR84) downregulates expression of TNF mRNA in tolerant human monocytes ${ }^{35}$. Consistent with this result, a defect in the capacity to activate nuclear factor $-\kappa B(N F-\kappa B)$ phosphorylation (which occurs downstream of TNF activity) was observed in patients with sepsis ${ }^{36}$. Of note, it is hypothesized that LPS tolerance establishes a form of innate immune memory that is adaptive against systemic infection and can be transmitted to daughter cells ${ }^{37}$.

\section{Central regulation}

The central nervous system (CNS) plays a major part in the dampening of the immune response after inflammation and infection ${ }^{38,39}$. The CNS controls inflammation by three main routes: sympathetic (or adrenergic) and parasympathetic (or cholinergic) pathways, which act regionally through innervation of lymphoid organs, and the hypothalamic-pituitary-adrenal axis, which acts at a systemic level through the release of glucocorticoids $^{39}$. For example, disruption of the vagal system in mice delays resolution of bacterial infections owing to decreased levels of peritoneal proresolving mediators and a dysregulated innate immune response (namely, a decreased number of group 3 innate lymphoid cells (ILC3s) and an increased number of macrophages) ${ }^{40}$. Sepsis is also suspected to induce long-term CNS microglia inflammation ${ }^{41}$ that might, in turn, maintain anti-inflammatory feedback loops. However, although there is no doubt about the role of the CNS in initiating a vigorous anti-inflammatory response in sepsis, the participation of each system or pathway remains to be examined in detail in sepsis-induced immunosuppression $^{42}$. An additional complexity in evaluating these mechanisms in patients with sepsis is that chemical agonists of these separate pathways are frequently used as therapies; for example, steroids and noradrenaline $e^{43}$.

\section{Metabolic reprogramming}

Immune cells use considerable amounts of energy for both their housekeeping functions and their specialized activities. For ATP generation, fuel molecules such as glucose are metabolized via glycolysis or oxidative phosphorylation (OXPHOS) ${ }^{44,45}$. OXPHOS, which is the major energy source in resting cells, is oxygen-dependent and occurs within the mitochondria. It comprises two reactions: first, conversion of intermediate molecules (pyruvate from glucose metabolism or fatty acids) to acetyl-CoA; and second, degradation of acetyl-CoA to $\mathrm{CO}_{2}$ in the tricarboxylic acid cycle. These reactions yield free electrons that are carried by electron transporters such as $\mathrm{NADH}$ to the electron transport chain located within the mitochondrial inner membrane. Ultimately, this process results in protons moving out of the mitochondrial matrix and in the establishment of a transmembrane electrochemical gradient that is harnessed for the production of ATP by ATP synthase. ATP production by OXPHOS is very efficient, with a total yield of 36 molecules of ATP from each molecule of glucose. Conversely, glycolysis occurs in the cytosol, where one molecule of glucose is broken down into two molecules of pyruvate. The net reaction is oxygen-independent and yields two molecules of ATP for each molecule of glucose. Pyruvate is then converted to lactate and generates the electron acceptor $\mathrm{NAD}^{+}$from NADH. Although ATP production by this pathway is not very efficient, it can be rapidly mobilized, in particular, through increased cell surface expression of the GLUT family of glucose transporters, which are initially stored intracellularly ${ }^{44,45}$. Whereas resting immune cells have low energy needs and derive most of their ATP from OXPHOS, activated leukocytes require a substantial increase in energy production and thus rely on the induction of glycolysis to carry out their functions. The shift from OXPHOS to glycolysis enables the cells to rapidly produce ATP. This phenomenon of metabolic reprogramming, termed the Warburg effect (that is, the generation of ATP by glycolysis even when adequate oxygen is available to oxidize glucose in the mitochondria), is a hallmark of cancer cells and of highly proliferating lymphocytes ${ }^{44,45}$.

At the molecular and intracellular levels, major metabolic and epigenetic reprogramming events leading to dramatic shifts in transcriptional profiles have emerged as central players in the induction and maintenance of sepsis-induced immune alterations ${ }^{46}$. Changes in the inflammatory capacity of monocytes are associated with altered metabolic processes; for example, the expression of the gene encoding hypoxia-inducible factor $1 \alpha(\mathrm{HIF} 1 \alpha)$ and other hypoxia-induced genes is upregulated in monocytes from patients with sepsis ${ }^{29}$. HIF1 $\alpha$ increases expression of IL-1 receptor-associated kinase M (IRAKM; also known as IRAK3), which reduces expression of inflammatory genes, thus linking metabolic changes to a tolerant state ${ }^{29}$. In addition, the distinct cytokine production profiles in human monocytes in the hyper-inflammatory state and those in the immunotolerant state after sepsis are associated with marked differences in cellular metabolism ${ }^{47}$. In particular, several metabolic defects have been described in patients with sepsis, including decreased glycolysis and oxygen consumption in monocytes ${ }^{48}$. These defects are associated with a dysfunctional mechanistic target of rapamycin (mTOR) pathway and were remedied by treatment with IFN $\gamma^{48}$. In accordance with these observations, the metabolic status of T lymphocytes in patients with sepsis is shifted towards a catabolic profile, with little reserve to promote effector functions and an inability to switch to an anabolic profile ${ }^{49}$. This altered metabolic status was associated with impaired effector functions (that is, proliferation) and marked alteration in mTOR pathway activation; moreover, these defects were remedied ex vivo by treatment with recombinant 
human IL-7 (rhIL-7) ${ }^{49}$. These last results are concordant with a study describing a hypometabolic profile in circulating lymphocytes, which was predictive of the development of secondary infections in patients after elective orthopaedic surgery ${ }^{50}$.

From a more general perspective, as a shift in metabolic profile is mandatory for immune cells to gain effector functions ${ }^{44}$, such metabolic alterations are probably involved in the development of sepsis-induced immune alterations. This metabolic shutdown might be similar to the cellular hibernation described in sepsis-induced organ failure ${ }^{51}$. Exploration of the immunometabolic pathways in sepsis thus represents an important area of research for the identification of novel targets to restore immune homeostasis after sepsis.

\section{Epigenetic regulation}

Epigenetic regulation is a key mechanism in the transcriptional reprogramming that occurs in a vast number of genes after sepsis ${ }^{52,53}$, among which are genes that are involved in the development of immune alterations ${ }^{54}$. Epigenetic regulation of transcription can occur through numerous mechanisms, but in general involves the regulated organization of gene loci into transcriptionally active or transcriptionally silent states. The major mechanisms of epigenetic change involve post-translational modifications to histone proteins in chromatin, such as methylation, acetylation, ubiquitylation and phosphorylation. Interestingly, epigenetic modifications can be passed from parent cells to daughter cells and thus have a central role in the potential long-term transmission of any shifted transcription profile across cell generations. Profound changes in histone methylation and acetylation as well as in DNA methylation have been described after sepsis ${ }^{55,56}$. In models of endotoxin tolerance, epigenetic modification of proinflammatory gene loci plays a prominent part in the regulation of their transcription ${ }^{34,57}$. Similar results were observed both in vitro in LPS tolerance experiments with human monocytic cell lines and in vivo in monocytes from patients with sepsis ${ }^{58}$. Thus, the 'cytokine storm of sepsis' may direct the loss of specific activating epigenetic marks at promoters of pro-inflammatory genes in monocytes and/or macrophages. Similarly, studies in lung-resident dendritic cells from mice after sepsis indicated that decreased methylation of histone H3 Lys4 (H3K4; a transcription-permissive mark) and increased methylation of H3K27 (a transcriptionrepressive mark) at the promoter region of the Ill2 gene correlated with decreased IL-12 production in response to Toll-like receptor (TLR) stimuli (that is, tripalmitoyl$S$-glyceryl cysteine (Pam3Cys), LPS and CpG-DNA) ${ }^{59}$. In the adaptive immune response, increased $\mathrm{H} 3 \mathrm{~K} 27$ methylation after sepsis occurs in $\mathrm{CD} 4^{+} \mathrm{T}$ cells at the promoter region of the gene encoding IFN $\gamma$ in T helper $1\left(\mathrm{~T}_{\mathrm{H}} 1\right)$ cells and the gene encoding GATAbinding factor 3 (GATA3) in $\mathrm{T}_{\mathrm{H}} 2$ cells ${ }^{60}$. Similarly, an increase in transcription-permissive histone modification of the Foxp3 locus might skew T cell differentiation towards the regulatory $\mathrm{T}\left(\mathrm{T}_{\text {reg }}\right)$ cell lineage after sepsis ${ }^{61}$.
These results are particularly important in light of the persistent nature of immunosuppression after sepsis, as these epigenetic modulations seem to remain long after sepsis has apparently resolved. Thus, these modifications might play an important part in shaping the immune response at time points long after recovery. It should be noted that other mechanisms of regulation, such as translational regulation, have been proposed to participate in the induction of immune alterations; for example, microRNAs and long non-coding RNAs have been implicated as players in additional post-transcriptional mechanisms that are involved in imparting LPS tolerance to cells ${ }^{26,62}$.

\section{Interplay between suppression mechanisms}

Numerous interactions between epigenetic, metabolic and transcriptional alterations have been observed in immune cells after sepsis ${ }^{46}$. One major example involves the deacetylase enzyme NAD-dependent protein deacetylase sirtuin 1 (SIRT1) - a metabolic sensor that is recruited to the promoters of inflammatory genes at late time points during the response to LPS ${ }^{63}$. During endotoxin tolerance, increased levels of SIRT1 accumulate at the promoters of the Tnf and Illb genes, which is dependent on the SIRT1 cofactor $\mathrm{NAD}^{+}$. This accumulation of SIRT1 leads to deacetylation of the transcription factor p65 (also known as RELA) at Lys310, which limits NF- $\kappa$ B-dependent transcription of these pro-inflammatory cytokine genes. In addition, sustained SIRT1 binding to promoters leads to recruitment of de novo-induced RELB, which directs assembly of the mature transcription repressor complex that further sustains endotoxin tolerance ${ }^{64}$. Therefore, the redox sensor SIRT1 coordinates an epigenetic, NF- $\kappa$ B-dependent RELA and RELB feedforward loop that regulates gene-selective changes during endotoxin tolerance. Of note, pharmacological SIRT1 activators can reduce inflammatory cytokine production in mice with early acute hyper-inflammatory sepsis, whereas SIRT1 inhibition during the post-acute hypo-inflammatory phase of sepsis reverses endotoxin tolerance and improves immunity ${ }^{63,65}$. Furthermore, beyond these interconnected mechanisms, it is important to note that sepsis occurs with very heterogeneous clinical presentations that are linked to the characteristics of each patient (genetic background, comorbidities, age and so on) or pathogen (type and site of infection, virulence), to the extent of cellular injury (release of damage-associated molecular patterns (DAMPs; also known as alarmins)) and to the effects of administered treatments (antibiotic therapy, blood transfusion, vasopressors or steroids; all of which are known to modulate immune functions). Thus, subtle combinations of these parameters probably have a role in the development of a stress-induced immune response (FIG. 1). Although the impact of each of these parameters in the development of the immune response during sepsis has been studied, thus far, no comprehensive systematic analysis has been undertaken to investigate the complex and intricate interplay between these parameters. This type of analysis is probably required to identify more efficient therapeutic targets that will lead to complete restoration of immune homeostasis in patients with sepsis. 


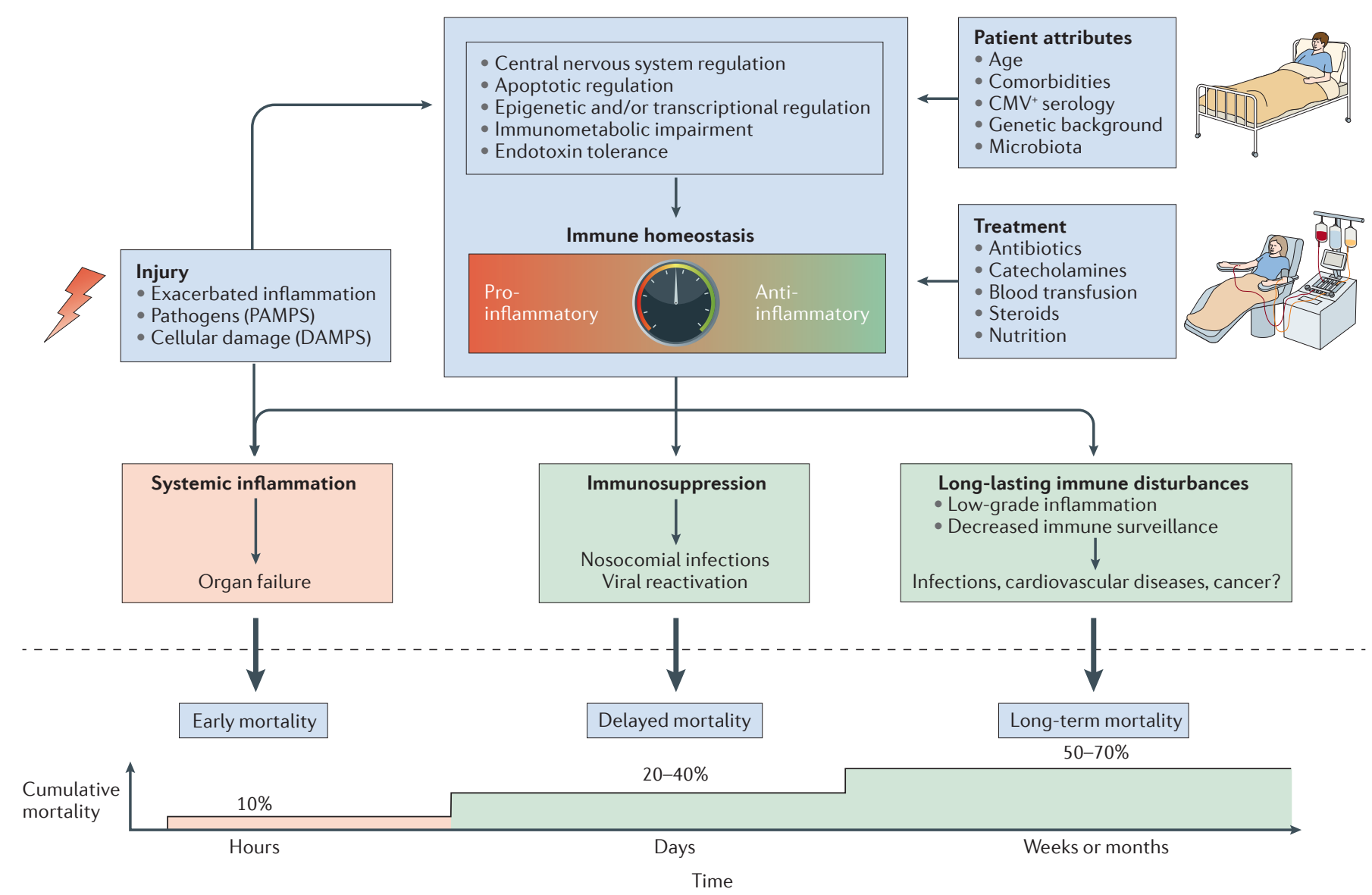

Figure 1 | Sepsis-induced immunosuppression mechanisms. Sepsis initiates a complex immune response that varies over time, with the concomitant occurrence of both pro-inflammatory and anti-inflammatory mechanisms. Mechanisms that regulate immune homeostasis during sepsis include central nervous system, apoptotic, epigenetic and/or transcriptional regulation in addition to immunometabolic impairment and endotoxin tolerance. Beyond these interconnected mechanisms, a subtle combination of parameters that is specific to each case of sepsis (including patient-specific and pathogen-specific characteristics, the extent of cellular injury and the types of treatment administered) also affects the regulation of immune homeostasis after sepsis. Altered immune regulation in patients with sepsis can lead to an exaggerated pro-inflammatory response, immunosuppression and/or long-lasting immune disturbances. Such dysregulated immune homeostasis has deleterious clinical consequences, such as organ failure, increased susceptibility to nosocomial and/or secondary infections, viral reactivations and/or increased mortality. CMV, cytomegalovirus; DAMPs, damage-associated molecular patterns; PAMPs, pathogen-associated molecular patterns.

\section{Altered immune functions}

Sepsis affects the innate immune system, including neutrophils, monocytes, dendritic cells and myeloid-derived suppressor cells (MDSCs) (FIG. 2).

\section{Immature neutrophils}

An increased proportion of immature neutrophils, which were identified phenotypically by their low expression of CD10 and CD16 and their immunosuppressive functions, is associated with increased early mortality after sepsis $^{66}$. Consistent with this observation, the existence of a subset of neutrophils $\left(\mathrm{CD} 16^{\text {hi }}, \mathrm{CD} \mathrm{L}^{\text {low }}\right)$ that are able to suppress $\mathrm{T}$ lymphocyte proliferation was proposed ${ }^{67}$. In general, profound alterations in the characteristics and functions of neutrophils were observed in patients during the first week after sepsis, including decreased chemotaxis, decreased oxidative burst and low lactoferrin content ${ }^{68}$. Again, an increased number of immature granulocytes (that is, $\mathrm{CD} 10^{\text {low }} \mathrm{CD} 16^{\text {low }}$ cells) is associated with an increased risk of death in these patients ${ }^{68}$. A similar association was also observed in patients with burns ${ }^{69}$. In patients with trauma, altered functional responses of neutrophils (after $\mathrm{N}$-formyl-methionylleucyl-phenylalanine (fMLP) challenge) was observed in patients who subsequently developed septic shock ${ }^{70}$. Accordingly, in a mouse model of sepsis, accumulation of high-mobility group protein B1 (HMGB1) in the late phase of sepsis participates in neutrophil defects (loss of oxidative burst) and thus might contribute to increased susceptibility to secondary infections ${ }^{71}$. In addition, in both patients and animals with sepsis, neutrophil overexpression of PDL1 (a ligand of the inhibitory co-receptor PD1 on T cells) is associated with deleterious outcomes ${ }^{72}$. These results in immature or immunosuppressive neutrophils are consistent with the expanding description of MDSCs after sepsis. 


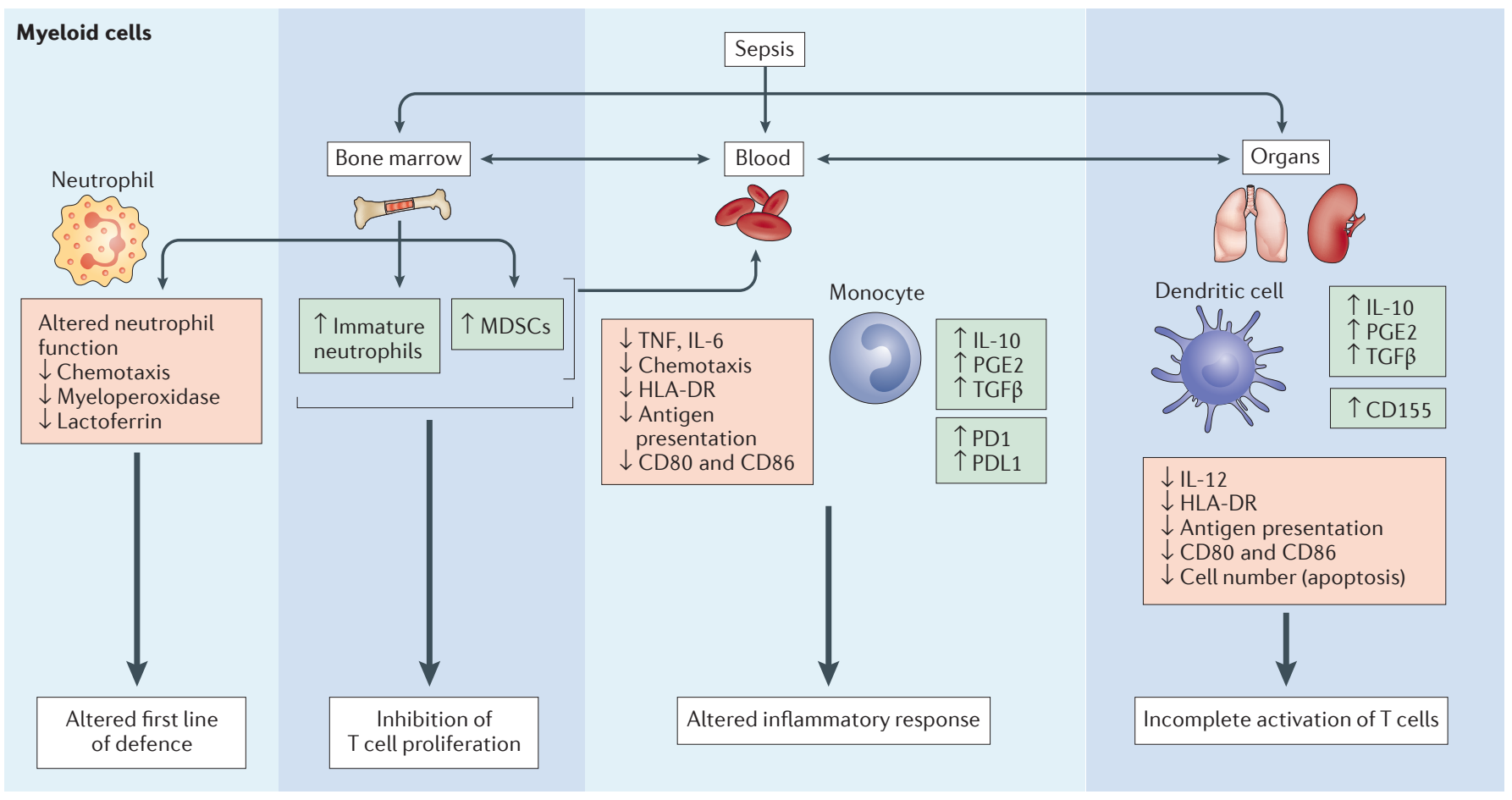

Figure 2 | Altered innate immune functions after sepsis. The innate immune response is altered after sepsis in patients and in mice. These immune alterations occur not only in the blood but also in the bone marrow and in organs that are distant from the initial site of infection. These alterations include increased release of immature neutrophils and myeloid-derived suppressor cells (MDSCs) from the bone marrow, which is also accompanied by alterations in the functions of mature neutrophils. The phenotype and functions of monocytes and/or macrophages and dendritic cells are also altered and include decreased effector functions, increased production of anti-inflammatory mediators and increased expression of immune checkpoint inhibitors. These alterations are deleterious for the host, as they result in a diminished first-line response against infection and decreased inflammation as well as altered T cell functions. HLA-DR, HLA class II histocompatibility DR; PD1, programmed cell death protein 1; PDL1, programmed cell death 1 ligand 1; PGE2, prostaglandin E2; TGF $\beta$, transforming growth factor- $\beta$; TNF, tumour necrosis factor.

\section{Myeloid-derived suppressor cells}

MDSCs constitute a heterogeneous population of immature myeloid cells that includes progenitors or precursors of monocytes, neutrophils and dendritic cells ${ }^{73}$. They are characterized mainly by their suppressive properties (on both innate and adaptive immunity) and are released following various inflammatory and/or infectious signals ${ }^{74}$. In experimental models of sepsis, the population of these cells is massively expanded ${ }^{75,76}$. Their increased abundance correlates with an increased abundance of $\mathrm{T}_{\text {reg }}$ cells ${ }^{77}$. In mice, different molecular mechanisms are proposed to direct a switch from differentiation of the usual neutrophil lineage to the generation of suppressor cells in the bone marrow ${ }^{78,79}$. MDSCs acquire their phenotype in the bone marrow and then migrate to lymph nodes to block lymphocyte proliferation in LPSimmunosuppressed mice ${ }^{80}$. Expansion of the MDSC population in mice with sepsis is under the control of TLR-dependent signalling ${ }^{81}$. The first results from studies of MDSCs in patients with sepsis are now available: increased numbers of MDSCs with documented suppression of lymphocyte proliferation were detected in patients with chronic immune suppression after severe sepsis or septic shock ${ }^{82,83}$. An association between an increased number of MDSCs in blood and future occurrence of nosocomial infections after sepsis has been described ${ }^{84}$. Unfortunately, a consensus on the definition of human MDSCs based on phenotypic characterization is currently lacking, and published results have been obtained using various phenotypic definitions, either from whole blood or from an MDSC-enriched fraction from Ficoll gradients $^{85}$. Thus, further clinical investigation will require better standardization of these cells.

Collectively, a strong rationale exists to reinvestigate the role of immature neutrophils and MDSCs in sepsis pathophysiology, especially in sepsis-induced immunosuppression. In particular, to date, no clinical studies have included sufficient numbers of patients to robustly assess the association between the response of immature neutrophils or MDSCs and deleterious outcomes (such as mortality, nosocomial infection rate and viral reactivation). Further studies are required in this regard.

\section{Monocytes}

The diminished capacity of innate immune cells (mainly monocytes) from patients with sepsis to release proinflammatory cytokines in response to LPS, TLR agonists or various bacterial compounds is probably the most 
prominent feature of sepsis-induced immunosuppression and illustrates the phenomenon of endotoxin tolerance (see earlier discussion). Many characteristics of human tolerant monocytes are recapitulated in the immunosuppressive M2 macrophage cell type. In the clinic, the magnitude and the persistence of this refractory state (mainly measured by decreased HLA-DR expression by monocytes as a surrogate marker) are largely associated with increased mortality and nosocomial infections ( $>150$ published articles $)^{86-88}$. Of note, PDL1 overexpression during monocyte anergy in sepsis has gained interest. As early as 2011, several groups reported an increase in the expression of a PD1-related molecule by leukocytes in patients with sepsis ${ }^{89,90}$. In addition, a multivariate analysis found that increased PDL1 expression by monocytes is an independent predictor of mortality after sepsis ${ }^{91}$. This analysis highlighted the importance of monocytes in the PD1 immunoregulatory system in sepsis-induced immune alterations. As is the case for neutrophils, subsets of monocytes belong to the heterogeneous group of MDSCs. Indeed, monocytic MDSCs (also known as mononuclear MDSCs) are defined as $\mathrm{CD} 14^{+} \mathrm{CD} 15^{-} \mathrm{HLA}-\mathrm{DR}^{-}$cells and by their suppressive effects on $\mathrm{T}$ cell responses ${ }^{84,92}$. An increased percentage of monocytic MDSCs has been observed in patients with sepsis ${ }^{84}$. In acute-on-chronic liver failure (a clinical condition with immune dysfunctions similar to sepsis), the population of these cells is massively expanded and is associated with increased mortality and secondary infections ${ }^{92}$. As for MDSCs, no consensus exists for the phenotyping of monocytic MDSCs. In addition, data from studies of monocytic MDSCs confirm that the widely reported reduction in HLA-DR expression by monocytes is a pivotal feature of monocyte anergy and is a reliable biomarker of sepsis-induced immunosuppression.

\section{Dendritic cells}

Decreased numbers of dendritic cells (in the spleen and blood) and reduced dendritic cell function have been widely reported in sepsis, in association with alterations of immune responses ${ }^{8,93}$. Both plasmacytoid and myeloid subsets of dendritic cells are substantially reduced by sepsis-induced apoptosis ${ }^{8,94,95}$. This cellular loss was more extensive in patients with sepsis who died than in those who survived ${ }^{94}$ or who developed secondary infections ${ }^{95}$. Importantly, normal dendritic cell counts recovered only weeks after sepsis in patients ${ }^{95}$. Accordingly, reduced dendritic cell function is still observed 6 weeks after sepsis in mice; this long-term effect is attributed to epigenetic alterations ${ }^{59,96}$. Altered functions of dendritic cells after sepsis are responsible for the inability of animals to mount an antigen-dependent response, owing to an absence of naive $\mathrm{T}$ cell activation. In these animals, treatment with FMSrelated tyrosine kinase 3 ligand (FLT3L; a growth factor for dendritic cells) restored dendritic cell functions and numbers ${ }^{97}$. In addition, blockade of sepsis-induced dendritic cell overexpression of CD155 (which is known to inhibit T cell functions through interaction with T cell immunoreceptor with immunoglobulin and ITIM domains (TIGIT)) protected mice from sepsis-induced mortality ${ }^{98}$. Thus, as key players in orchestrating immune responses, dendritic cells have a pivotal role in the development of sepsis-induced immunosuppression ${ }^{93,99}$. This role is confirmed by the observation that, after pneumonia, the cellular and cytokine environment in the lung induced a shift in dendritic cells towards a tolerogenic profile that predisposed animals to secondary infections in the long term, a phenomenon that is driven by transforming growth factor- $\beta$ (TGF $\beta$ ) and $\mathrm{T}_{\text {reg }}$ cell induction ${ }^{100}$.

\section{Lymphocytes}

In addition to its effects on cells of the innate immune system, sepsis also alters the function and composition of cells involved in acquired immunity (FIG. 3). A major characteristic of the adaptive immune response after sepsis is the tremendous lymphopaenia that occurs in patients with sepsis ${ }^{101}$. This apoptotic process affects every lymphocyte subpopulation, is present at patient admission and persists at least until the end of the first week after admission ${ }^{101,102}$. Retrospective studies have shown that persistent lymphopaenia is associated with an increased risk of death and nosocomial infections in patients with sepsis ${ }^{103-105}$.

Innate-type lymphocytes. In addition to their roles in the initial inflammatory response and in combating infection, innate-type lymphocytes, such as natural killer (NK) cells, innate lymphoid cells (ILCs), natural killer T (NKT) cells or mucosal-associated invariant T (MAIT) cells, are also affected by sepsis and may participate in immunosuppression. In particular, a phenomenon similar to endotoxin tolerance affects NK cells after sepsis ${ }^{106}$. NK cell immunosuppression was also observed in patients with brain injuries ${ }^{107}$. Besides reduced cytokine production, NK cell cytotoxic activity is also reduced in patients with sepsis ${ }^{108}$. In mice, NK cell tolerance to pathogen-associated molecular patterns (PAMPs) was reversed by depletion of $\mathrm{T}_{\text {reg }}$ cells ${ }^{106}$. A dynamic inverse correlation between increased $\mathrm{T}$ cell immunoglobulin mucin receptor 3 (TIM3; also known as HAVCR2) expression and reduced IFN $\gamma$ production in NK cells was shown in mice with sepsis ${ }^{109}$, whereas TIM3 blockade improved the IFN $\gamma$ production capacity of NK cells. Similarly, expression of PD1 and PDL1 was markedly increased in NK and NKT cells in mice with sepsis, whereas inhibition of PD1 or PDL1 signalling caused a twofold increase in survival of mice with sepsis ${ }^{110}$. This immunosuppressed NK cell response is deleterious to the host, as impaired NK cell function precedes the occurrence of cytomegalovirus (CMV) reactivation in critically ill patients ${ }^{111}$. Only a few studies have evaluated the responses of ILCs, NKT cells or MAIT cells in sepsis-induced immunosuppression. Hepatic immature NKT cells have a role in injuryinduced immunosuppression through central regulation with noradrenergic innervation ${ }^{112}$. Interestingly, rhIL-7 immunotherapy ameliorates sepsis-induced loss of lung ILCs in mice ${ }^{113}$. Finally, in patients with severe bacterial infections, similarly to NKT cell and $\gamma \delta$ T cell number, MAIT cell number is substantially decreased, and persistent diminution of MAIT cell number is associated with increased incidence of intensive care unit (ICU)-acquired infections ${ }^{114}$. 

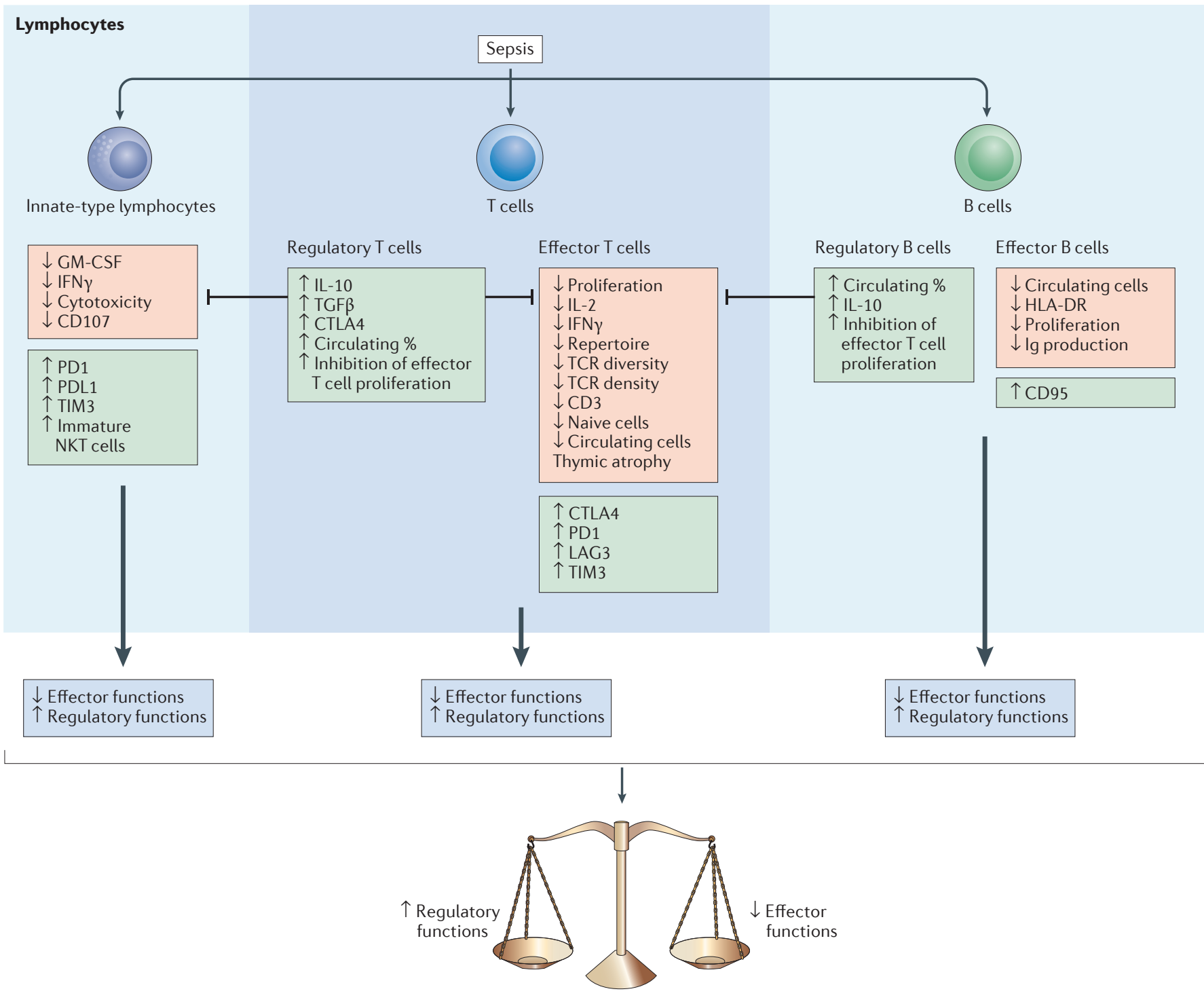

Figure 3 | Altered adaptive immune functions after sepsis. The adaptive immune response is altered after sepsis in patients and in mice. Sepsis affects the effector functions and phenotypes of T cells, B cells and innate-type lymphocytes, such as natural killer (NK) cells. Cytotoxicity in NK cells, IFN $\gamma$ production in T lymphocytes and cellular proliferation in B lymphocytes are reduced, whereas the expression of immune checkpoint inhibitors is increased in NK cells and T lymphocytes. The proportions of exhausted and regulatory T cells and B cells are elevated after sepsis, when these cell types play a major part in regulating effector lymphocyte functions. CTLA4, cytotoxic T lymphocyte protein 4; HLA-DR, HLA class II histocompatibility DR; Ig, immunoglobulin; GM-CSF, granulocyte-macrophage colony-stimulating factor; LAG3, lymphocyte activation gene 3 protein; NKT, natural killer T; PD1, programmed cell death protein 1; PDL1, programmed cell death 1 ligand 1 ; TCR, T cell receptor; TGF $\beta$, transforming growth factor- $\beta$; TIM3, T cell immunoglobulin mucin receptor 3.

B lymphocytes. B lymphocytes carry out multiple functions in sepsis. In patients and in mice, B cells first play a part in the major pro-inflammatory immune response that occurs after infection ${ }^{115}$. Indeed, induction of sepsis in B cell-deficient mice leads to increased mortality, whereas treatment of $\mathrm{V}(\mathrm{D}) \mathrm{J}$ recombination-activating protein 1 (RAG1)-deficient mice with B cells markedly improves survival during sepsis ${ }^{116,117}$. Subpopulations of $\mathrm{B}$ cells might have specific roles in this context. For example, innate response activator (IRA) B cells are induced after sepsis in mice, their activity is dependent on pattern recognition receptors, and they initiate an antimicrobial response through the production of GM-CSF ${ }^{117}$. In addition, IL-3-producing IRA B cells induce emergency myelopoiesis and potentiate septic shock in mice as well as in patients with sepsis ${ }^{118}$. Innate functions for B1a type B cells after sepsis have also been described ${ }^{119}$. Conversely, the phenotype and function of B cells during the immunosuppressive phase of sepsis has scarcely been studied, although subpopulations of B cells with regulatory properties have been described in other contexts ${ }^{120}$. In patients and in mice, several studies found a marked 
decrease in B cell number owing to increased apoptosis ${ }^{101}$. Changes in the proportions of B cell subsets have been observed in patients with sepsis, with higher apoptosis in memory B cells than in other types of B cells and a potentially important role for extracellular signal-regulated kinase (ERK)-activation-associated B cell death in sepsis $^{121}$. Sepsis also triggers a prolonged reduction in the number of naive B cells and increases B cell exhaustion in the periphery in patients with sepsis ${ }^{122}$. These effects are associated with an impairment in the capacity of B cells to produce IgM but not IgG, as described previously ${ }^{123}$. The percentage of $\mathrm{CD} 19^{+} \mathrm{CD} 24^{\text {high }} \mathrm{CD} 38^{\text {high }} \mathrm{B}_{\text {reg }}$ cells was elevated in neonatal patients with sepsis compared with that in healthy individuals ${ }^{124}$. Through the study of the expression of several surface markers on B cells (HLA-DR, maturation marker and a $\mathrm{CD} 95^{\text {hi }} \mathrm{CD} 21^{\text {low }}$ phenotype) and the assessment of B cell functionality (activation response, proliferation and IL-10 production), we recently provided several arguments suggesting that, as observed previously for T lymphocytes, the B cell response is altered during sepsis-induced immunosuppression and is oriented towards an immunoregulatory or exhausted profile (C.-A. Gustave, M. Gossez, J. Demaret, T. Rimmelé, A. Lepape, C. Malcus, F. Poitevin-Later, L. Jallades, J. Textoris, G.M. and F.V., unpublished observations). Exhausted B cells may thus represent a complementary pathway of immune regulation after septic shock, although further studies are necessary to demonstrate this aspect.

T lymphocytes. $\mathrm{CD} 4^{+} \mathrm{T}$ cells and $\mathrm{CD} 8^{+} \mathrm{T}$ cells that do not undergo apoptosis after sepsis display an anergic or exhausted profile, with major defects in cell phenotype and functions. This profile includes a shift towards decreased IL- 2 and IFN $\gamma$ production capacity and decreased $\mathrm{T}$ cell proliferation ${ }^{8,125-127}$. Both intrinsic mechanisms, which directly affect $T$ cells, and indirect mechanisms, which work through altered antigen-presenting cell or immature neutrophil functions, might participate in the pathophysiology of $\mathrm{T}$ cell alterations. T cell-intrinsic defects are probably induced by initial massive activation after sepsis, as increased expression of activation markers such as glucose transporter type 1, erythrocyte/brain (GLUT1; also known as SLC2A1), basal signal transducer and activator of transcription 5 (STAT5) phosphorylation or CD69 on circulating T cells after sepsis has been observed ${ }^{49,128,129}$. Epigenetic reprogramming of $\mathrm{T}$ cells has also been described in mice with sepsis, with histone methylation and chromatin remodelling observed within the promoter regions of the genes encoding T-box protein 21 (TBX21; also known as TBET) and GATA3, thereby contributing to lymphocyte anergy ${ }^{60}$. In patients with sepsis, miR-31, a regulator of the transcription factors HIF1 $\alpha$ and NF- $\kappa B$, is a post-transcriptional regulator of sepsis-associated $\mathrm{T}$ cell alterations ${ }^{130}$. In addition, increased expression of co-inhibitory receptors such as PD1, cytotoxic T lymphocyte antigen 4 (CTLA4), TIM3, lymphocyte activation gene 3 protein (LAG3), TNFrelated apoptosis-inducing ligand (TRAIL; also known as TNFSF10) or B and T lymphocyte attenuator (BTLA) has been repeatedly described in T cells in both mice and patients with sepsis ${ }^{89,90,127,128,131}$. This increased expression participates in T cell alterations, as co-inhibitory receptor blockade improves $\mathrm{T}$ cell functions after sepsis (see below). T cell receptor (TCR) diversity is markedly reduced after septic shock in patients in association with increased risk of nosocomial infections ${ }^{132}$. Metabolic reprogramming also participates in $\mathrm{CD} 4^{+} \mathrm{T}$ cell dysfunction after sepsis. We recently showed that basal metabolic content was altered in T lymphocytes from patients with sepsis in association with a decreased concentration of ATP and with inhibition of the OXPHOS and glycolysis pathways. Upon ex vivo stimulation, T lymphocytes from patients with sepsis failed to induce glycolysis, OXPHOS, ATP production, GLUT1 expression, glucose entry into cells and $\mathrm{T}$ cell proliferation to levels similar to those in healthy individuals. This phenotype was associated with decreased activation of mTOR (but not of AKT or HIF1 $\alpha$ ) and only a minor decrease in the phosphorylation of 5'-AMP-activated protein kinase catalytic subunit- $\alpha$ (AMPKa), which could be restored by rhIL-7 (REF. 49).

Regulatory $T$ cells. The proportion of $\mathrm{CD}^{+} \mathrm{CD} 25^{+} \mathrm{CD} 127^{\text {low }} \mathrm{FOXP}^{+}\left(\mathrm{CD} 39^{+}\right) \mathrm{T}_{\text {reg }}$ cells is elevated after sepsis in mice and in patients ${ }^{133-136}$, which might participate in decreasing $\mathrm{T}$ cell proliferation ${ }^{133}$. After recovery from severe sepsis, mice have persistently increased $\mathrm{T}_{\text {reg }}$ cell activity, which impairs the antitumour response that is mediated by $\mathrm{CD} 8^{+} \mathrm{T}$ cells and thus favours tumour growth ${ }^{61}$. In addition, endogenous IL-33, which is released in response to severe tissue damage, has an essential function in the expansion of $\mathrm{T}_{\text {reg }}$ cells after sepsis and in the development of long-term immunosuppression. IL-33 activates group 2 innate lymphoid cells (ILC2s), which produce IL-4 and IL-13; these cytokines drive M2 polarization of macrophages, resulting in the expansion of $\mathrm{T}_{\text {reg }}$ cells via IL-10 production $^{137}$.

Immunosuppression in lymphoid organs. Pioneering autopsy studies of adults who died of sepsis showed substantial apoptosis-induced loss of cells of the adaptive immune system in the spleen, blood and gut-associated lymphoid tissues ${ }^{12,138}$. Similar results were observed in children with sepsis-induced multiple organ failure ${ }^{139}$. The ubiquity of apoptosis in the blood, lungs, spleen and bone marrow during sepsis was demonstrated in murine models of sepsis ${ }^{17}$. In 2011, a convincing postmortem study of patients who died of sepsis reported that most phenotypic and functional alterations in the blood of patients (which were previously described by several groups) were also present in organs distant from the initial infection ${ }^{127}$. In a few studies, patients with initial extrapulmonary infections showed an association between decreased monocyte HLA-DR expression and elevated IL-10 levels in bronchoalveolar lavage fluid and secondary pulmonary infections ${ }^{140,141}$. In addition, decreased monocyte HLA-DR expression was detected in the bone marrow of patients with sepsis ${ }^{142}$. This result is consistent with studies of humanized mice showing the expansion of the primitive haematopoietic stem and progenitor cell (HSPC) population in the bone 
marrow, with a concomitant decrease in the population of the more committed progenitor cells ${ }^{143}$. This effect was dependent on Notch signalling and STAT3 (which has an established role in emergency granulopoiesis under GM-CSF control) and required TLR engagement. These researchers hypothesize that the activation of Notch in HSPCs serves as a protective mechanism against exhaustion during severe infections but consequently might also alter the maturation of HSPCs into differentiated progenitors with efficient functions ${ }^{143,144}$. This hypothesis is consistent with the observations of immature neutrophils and MDSCs discussed earlier. Early T cell lineage progenitor differentiation is consistently inhibited in the bone marrow after sepsis ${ }^{143}$ in association with a loss of thymocytes due to apoptosis ${ }^{145}$. Thymic atrophy in mice with sepsis has been described ${ }^{146,147}$ together with altered expression of chemokine receptors, which leads to inefficient homing capacity of $\mathrm{T}$ cells ${ }^{146}$. Thus, the altered $\mathrm{T}$ cell maturation after sepsis occurs in both the bone marrow and the thymus.

Collectively, these data show that, in addition to circulating cells, systemic immunosuppression affects both primary and secondary lymphoid organs after sepsis and, as such, immunosuppression can be considered an additional example of long-lasting organ failure in sepsis.

\section{Clinical consequences Infections and long-term mortality}

Increasing clinical evidence suggests that patients with sepsis are incapable of fighting primary bacterial infections and secondary infections by weakly virulent pathogens. For example, a postmortem study showed that approximately $80 \%$ of patients with sepsis still presented with unresolved septic foci at the time of death, despite appropriate antibiotic therapy ${ }^{148}$. Secondary infections due to microorganisms that are usually only pathogenic in immunocompromised hosts (Acinetobacter baumannii, Pseudomonas aeruginosa and Enterococcus spp.) are frequently observed in patients with sepsis ${ }^{149-151}$. In addition, systemic fungal infections (candidiasis, aspergillosis and mucormycosis) have become a prominent cause of complications in patients with severe injuries ${ }^{152,153}$. The reactivation of dormant viruses (for example, torque teno virus and herpes simplex virus) in patients in the ICU is also gaining much attention ${ }^{154,155,156}$. A large body of literature exists describing the reactivation of CMV in patients as a consequence of a dampened immune defence. Indeed, frequent reactivation of CMV occurs in patients with sepsis in association with a marked increase in mortality ${ }^{157-159}$. Of note, CMV seroprevalence, by itself, is a risk factor of poor outcome in patients in the $\mathrm{ICU}^{160}$. The importance of CMV reactivation has been demonstrated by its considerable confounding effect in clinical trials of patients with sepsis ${ }^{161}$.

The increased susceptibility of patients with sepsis to secondary infections is consistent with data on hospital readmission rates and long-term mortality after sepsis. Several studies have reported that approximately $25 \%$ of patients who survive sepsis are readmitted to the hospital within 30 days after their first hospital discharge $\mathrm{e}^{162-165}$.
In these patients, infection was the leading cause of additional hospitalization ${ }^{166-168}$. In addition, this higher susceptibility to recurrent infections in patients with sepsis can persist for an entire year following the first episode of sepsis, and increased long-term mortality (in the 10 years following the initial episode) has been described in patients who survived sepsis ${ }^{167,169,170}$. Although clinical demonstration is still lacking, these data suggest that patients with sepsis have persistent immune defects on a long-term basis after sepsis, which might contribute to their increased susceptibility to subsequent infections.

\section{PICS}

Many researchers have proposed that, in addition to long-lasting immunosuppression, persistent low-grade inflammation (maintained by the release of DAMPs, such as HMGB1 or S100A8 and S100A9) may be present in patients with sepsis after hospital discharge and could contribute to a compensatory anti-inflammatory or immunosuppressive mechanism in which MDSCs might play a pivotal part ${ }^{171,172}$. This persistent inflammation is associated with long-term catabolism and malnutrition, which are also frequently observed in chronic inflammatory diseases ${ }^{171,173}$. This chronic illness has been termed persistent inflammation, immunosuppression and catabolism syndrome (PICS) and is associated with impaired wound healing and increased risk of subsequent infections. This hypothesis is consistent with the increased risk of infections and subsequent mortality in survivors of sepsis and with the description of persistent signs of immune alterations and low-grade inflammation in hospitalized patients with sepsis or at ICU discharge. These alterations include increased MDSC abundance ${ }^{82}$, lack of recovery of monocyte HLA-DR expression ${ }^{174}$ and increased IL- 6 concentration ${ }^{175}$. However, as mentioned previously, almost no data are available regarding the immune status of patients with sepsis upon hospital discharge, and when available, such data (based on small cohorts) indicate the absence of persistent immune alterations or inflammation in these patients ${ }^{174,176,177}$. Thus, at present, the PICS concept mostly applies to patients with long hospital stays, and long-term immune status after sepsis remains to be evaluated.

\section{Sepsis accelerates immunosenescence}

The immune characteristics of PICS (that is, increased C-reactive protein (CRP) levels and lymphopaenia) closely resemble the features of immunosenescence. Indeed, immunosenescence is associated with a shift towards increased differentiation of myeloid progenitors with MDSC characteristics in the bone marrow (at the expense of lymphoid progenitors), lymphopaenia (with a decreased proportion of naive T cells), decreased $\mathrm{T}$ cell repertoire, thymic involution, chronic low-grade inflammation and telomere attrition ${ }^{178,179}$. Sepsis-induced immune alterations could thus be considered accelerated immunosenescence, suggesting that survivors of sepsis have a reduced functional immune response in subsequent years. In this sense, rapidly restoring immune homeostasis after sepsis might provide long-term protective effects that are the result of a fully functional 
immune system. Thymus regeneration is the subject of intense research in elderly patients or in those who have undergone bone marrow transplantation. Some candidate molecules for thymus regeneration are already in use (for example, IL-7) or will be tested for the treatment of sepsis in the near future ${ }^{179,180}$.

As discussed earlier, further investigation of the longterm immune alterations that occur after sepsis is highly desirable. Indeed, it is unclear whether lingering inflammation after sepsis is a reflection of inadequate initial control of an infection (a hypothesis supported by a study of postmortem biopsy samples ${ }^{148}$ ), which leads to prolonged DAMP release or to an inability of the host to control the initial inflammatory response due to, for example, sustained CNS stimulation ${ }^{41}$.

\section{Therapeutic promise of immunostimulation The future for anti-inflammatory strategies}

Despite the failure of numerous clinical trials, some promise likely still exists in using anti-inflammatory treatment strategies in the early hours after the onset of sepsis. Indeed, retrospective studies showed that subgroups of patients with sepsis might benefit from antiinflammatory treatment strategies such as IL-1 receptor blockade ${ }^{181}$ or anti-TNF treatments ${ }^{182}$. A meta-regression analysis of $>20$ randomized controlled trials that tested various anti-inflammatory molecules for the treatment of sepsis indicated that these approaches are probably more effective in patients with the most severe sepsis ${ }^{183}$, whereas these approaches are potentially deleterious in patients with lower risk of death (that is, in patients with less severe sepsis). Thus, a major challenge is to identify patients who could benefit from anti-inflammatory treatments. In addition, to avoid potentially deleterious effects, anti-inflammatory treatments should be administered for a short period of time after admission (for example, for less than 2 days) or halted upon catecholamine vasopressor withdrawal in patients with septic shock. To aid in formulating treatments, adaptable randomized controlled trial designs could be envisaged. For example, anti-inflammatory treatments in the first hours after sepsis could be combined with immunostimulation after $72 \mathrm{~h}$ if the presence of immunosuppression is confirmed by immune monitoring. Avoiding use of the ' 28 -day mortality' statistic in randomized controlled trials is also desirable, as long-term outcomes as well as nosocomial infection rate, numbers of ventilation-free days, hospitalization duration or medico-economic aspects should be considered.

\section{Preclinical models}

The goal of immunostimulation is to revitalize the immune system to allow clearance of initial infectious foci or to fight secondary infections. The preclinical twohit model of sepsis suitably recapitulates this clinical condition of consecutive infections. In this model, animals undergo low-severity CLP and then receive antibiotics, analgesics and fluid resuscitation, and for the most part, they survive this initial infectious challenge (as do most patients in the ICU). Nevertheless, after a few days, these mice present with features of immunosuppression and are unable to control a second infectious challenge (for example, P. aeruginosa, Aspergillus spp., Staphylococcus aureus and Candida albicans) that mimics nosocomial infections in patients ${ }^{113,184,185}$. This second hit kills the majority of animals with sepsis in a few days, whereas sham-CLP animals easily eradicate such infections, with almost no mortality. In this model, prophylactic use of immunostimulant molecules (IL-7, FLT3L, anti-PD1 or anti-PDL1 antibodies) administered to mice before the second hit improves survival and the ability to fight secondary infections. Similar effects were obtained by injecting immunocompetent cells from healthy animals into mice with sepsis ${ }^{184}$. Of note, inoculation of mice with malignant tumour cells is also used as a second challenge. In immunosuppressed mice subjected to CLP, tumour cells grow rapidly, whereas their growth is controlled in sham-CLP animals ${ }^{61,81}$.

\section{Similarities with cancer}

Cancer and sepsis induce a similar weakening of the immune system, and many observations made in sepsis can be transferred to cancer and vice versa ${ }^{186,187}$. Indeed, the development of cancer and chronic infections (for example, from HIV or hepatitis $\mathrm{C}$ virus) are favoured by a variety of immune subversion mechanisms, such as the production of anti-inflammatory cytokines, induction of $\mathrm{T}_{\text {reg }}$ cell and MDSC differentiation and overexpression of inhibitory co-receptors - all characteristics seen in sepsis (TABLE 1). Considering the medical revolution of immunotherapies in treating cancer, it is possible that the treatment of sepsis-induced immunosuppression may benefit from progress in cancer immunotherapy in the forthcoming years. The transfer of anti-PD1 antibodies from cancer to sepsis is an illustrative example (see below). In line with this proposal, an obvious next therapeutic option lies in the field of pattern recognition receptors, which constitute immunoadjuvant treatments to improve cancer immunotherapy ${ }^{188}$. Currently, $>40$ clinical trials are assessing the efficacy of several TLR agonists in the treatment of cancer ${ }^{188}$. This approach has already shown success in two-hit models of injury-induced immunosuppression (from trauma and $S$. aureus infection or CLP and P. aeruginosa infection) in which activation of TLRs improved mortality ${ }^{189,190}$. Of note, also in line with cancer therapy, it should be remembered that intrapulmonary $\operatorname{Tn} f$ gene expression locally reversed sepsis-induced immunosuppression and improved survival in mice subjected to CLP ${ }^{191}$.

\section{Current immunostimulation trials}

As persistent sepsis-induced immunosuppression is associated with deleterious outcomes, immunostimulation seems a reasonable therapeutic option, especially because our capacity to treat patients aggressively with supportive therapy (in parallel with therapy to control the infection source) during the very first hours of shock has improved. To date, most patients now survive this critical stage (that is, early mortality has decreased) but eventually die later during a state of immunosuppression. Various immunostimulant strategies have already been evaluated in sepsis. Thymosin $\alpha 1$ seems promising ${ }^{192}$, and 
a randomized controlled trial is underway in China ${ }^{193}$. In addition, other immunoadjuvant therapies are under investigation (IL-15, IL-3, FLT3L, anti-LAG3 antibodies, anti-TIM3 antibodies, anti-CTLA4 antibodies, adrenergic blockers and anti-IL-10 antibodies) $)^{3,7,8,43,56,172,194}$, although none of them have been as well studied as the four molecules discussed below.

IFN $\gamma$. IFN $\gamma$ is a prototypical cytokine produced by $\mathrm{T}_{\mathrm{H}} 1$ cells and is a major activator of monocytes, increasing their antigen-presentation capacity and phagocytic properties; IFN $\gamma$ primes monocytes for the LPS-induced release of inflammatory cytokines. The beneficial effect of IFN $\gamma$ on monocyte deactivation in patients with sepsis was first described in a limited open-label study in 1997 (REF. 15). A study in 2002 confirmed these results in patients with trauma by showing that inhaled IFN $\gamma$ treatment led to recovery of HLA-DR expression in alveolar macrophages and decreased incidence of ventilatorassociated pneumonia ${ }^{195}$. Following these two seminal studies, the use of IFN $\gamma$ in patients with severe infection has only been reported in clinical cases ${ }^{196,197}$. These case reports systematically found an association between this immune-adjuvant treatment and improved immune functions, including increased HLA-DR expression. IFN $\gamma$ therapy was even proposed to improve outcomes and immune dysfunctions in invasive fungal infections ${ }^{198}$. Interestingly, a case report of a woman with fungal sepsis (mucormycosis) who was unresponsive to conventional therapy showed the effectiveness of IFN $\gamma$ therapy in combination with nivolumab (an anti-PD1 antibody) in restoring immune functions and clearing the invasive infection ${ }^{199}$. Despite these promising preliminary data, no randomized controlled trials have tested IFN $\gamma$ therapy

Table 1 | Sepsis and cancer: common immunosuppressive mechanisms

\begin{tabular}{|c|c|c|}
\hline $\begin{array}{l}\text { Immune } \\
\text { source }\end{array}$ & Alterations & $\begin{array}{l}\text { Immunoadjuvant } \\
\text { therapies }\end{array}$ \\
\hline Myeloid cells & $\begin{array}{l}\text { - } \uparrow \text { Immature neutrophils } \\
\text { - } \uparrow \text { MDSCs } \\
\text { - } \downarrow \text { mHLA-DR expression } \\
\text { - } \uparrow \text { Tolerogenic dendritic cells }\end{array}$ & $\begin{array}{l}\text { - GM-CSF } \\
\text { - TLR agonists* } \\
\text { - FLT3L* } \\
\text { - TNF* }\end{array}$ \\
\hline $\begin{array}{l}\text { Lymphoid } \\
\text { cells }\end{array}$ & $\begin{array}{l}\text { - } \downarrow \text { Cytokine production in T lymphocytes } \\
\text { (IL-2, IFN } \gamma \text { ) } \\
\text { - Altered metabolism } \\
\text { - } \downarrow \text { Proliferation } \\
\text { - Exhausted phenotype with increased } \\
\text { expression of immune checkpoint } \\
\text { inhibitors (PD1, TIM3, LAG3) } \\
\text { - Altered NKT cells } \\
\text { - } \uparrow \text { Proportion of T } \text { Treg }_{\text {rells and } B_{\text {reg }} \text { cells }} \\
\text { - CD155 overexpression }\end{array}$ & $\begin{array}{l}\text { - Anti-PD1 antibodies } \\
\text { - Anti-PDL1 antibodies } \\
\text { - Anti-CTLA4, anti-TIM3 } \\
\text { and anti-LAG3 antibodies* } \\
\text { - rhlL-7, IL-15* } \\
\text { - Thymosin a1 }\end{array}$ \\
\hline $\begin{array}{l}\text { Systemic } \\
\text { cytokine } \\
\text { release }\end{array}$ & $\begin{array}{l}\text { - } \uparrow \text { IL-10 } \\
\text { - } \uparrow \text { PGE2 } \\
\text { - } \uparrow \mathrm{TGF} \beta\end{array}$ & $\begin{array}{l}\text { - GM-CSF } \\
\text { - TLR agonists* } \\
\text { - FLT3L* } \\
\text { - TNF* }\end{array}$ \\
\hline
\end{tabular}

$B_{\text {reg }}$ cells, regulatory B cells; CTLA4, cytotoxic T lymphocyte protein 4; FLT3L, FMS-related tyrosine kinase 3 ligand; GM-CSF, granulocyte-macrophage colony-stimulating factor; LAG3, lymphocyte activation gene 3 protein; MDSCs, myeloid-derived suppressor cells; mHLA-DR, monocytic HLA class II histocompatibility DR; NKT, natural killer T; PD1, programmed cell death protein 1; PDL1, programmed cell death 1 ligand 1; PGE2, prostaglandin E2; rhIL-7, recombinant human IL-7; TGF $\beta$, transforming growth factor $-\beta ;$ TIM3, T cell immunoglobulin mucin receptor 3; TLR, Toll-like receptor; $T N F$, tumour necrosis factor; $\mathrm{T}_{\text {reg }}$ cells, regulatory T cells. ${ }^{*}$ Only tested in experimental models of sepsis. in patients in the ICU. Of note, expert recommendations propose IFN $\gamma$ as a therapeutic alternative for treating non-resolving fungal infections in immunosuppressed patients with haematological cancers ${ }^{200}$.

Granulocyte-macrophage colony-stimulating factor. GM-CSF is a haematopoietic growth factor that stimulates the production of neutrophils and monocytes by the bone marrow. This cytokine also potently stimulates the activation and survival of monocytes. In an open-label randomized controlled trial of children without neutropenia who were admitted to a paediatric ICU with immunoparalysis (that is, reduced ex vivo TNF production), GM-CSF therapy facilitated recovery of the TNF response and completely prevented nosocomial infections ${ }^{201}$. In a case report of four patients, GM-CSF therapy was associated with reversal of negative skin testing and with a substantial decrease in recurrent infections in otherwise immunologically healthy children with recurrent infections ${ }^{202}$. Finally, in 56 neonates with sepsis, GM-CSF treatment resulted in an earlier increase in the number of monocytes expressing HLA-DR following the onset of sepsis ${ }^{203}$. In adults, in a case of non-resolving life-threatening legionellosis, GM-CSF administration improved monocyte function, accelerated bacterial clearance and probably contributed to recovery, with no adverse effects ${ }^{204}$. The most convincing results were observed in a randomized controlled trial of 39 patients with sepsis who had immune dysfunctions (on the basis of decreased monocyte expression of HLA-DR): biomarker-guided GM-CSF therapy in these patients was safe and effectively restored the immunocompetence of monocytes ${ }^{205}$. In addition, GM-CSF treatment shortened the time of mechanical ventilation and the duration of hospital or ICU stay ${ }^{205}$. Overall, although no evidence of short-term survival benefit from GM-CSF treatment exists, studies of biomarker-guided selection of patients suggests GM-CSF therapy markedly improves clinical end points (faster recovery, decreased duration of hospital stay, earlier ventilator weaning and decreased medical costs) without deleterious adverse effects ${ }^{206}$. GM-CSF thus represents a promising immunoadjuvant therapy in patients with sepsis, although larger randomized controlled trials are now warranted to confirm these initial results. The GRID trial is a French multicentre academic clinical trial evaluating this therapeutic approach in patients with septic shock ${ }^{207}$, the results of which are expected to be published in 2018 .

IL-7. IL-7 is produced by bone marrow and thymus cells and is a cytokine that is indispensable for the growth, differentiation and effector functions of T cells, whereas IL-7 negatively regulates $\mathrm{T}_{\text {reg }}$ cells ${ }^{180,208,209}$. rhIL-7 has been proposed for the treatment of patients with lymphopaenia and lymphopaenia-driven diseases ${ }^{208}$. This cytokine is also currently undergoing clinical testing as an immune-enhancing agent in patients with cancer or progressive multifocal leukoencephalopathy due to John Cunningham (JC) virus infection after allogeneic bone marrow transplantation, and is hypothesized to rejuvenate immune functions in elderly individuals ${ }^{179,180,208,209}$. 
As patients with sepsis present with severe lymphocyte alterations that are associated with increased mortality, rhIL-7 therapy is recommended for these patients and is even considered by experts to be one of the most promising potential adjuvant treatments for sepsis-induced immunosuppression ${ }^{3}$. Indeed, results from preclinical studies, both in vivo in mice and ex vivo in cells from patients with sepsis, seem quite compelling. In a murine two-hit model of sepsis, rhIL-7 treatment reduced apoptosis of $\mathrm{T}$ cells, restored IFN $\gamma$ production, improved leukocyte migration to sites of infection, facilitated pathogen clearance (bacterial or fungal) and improved the survival of mice with sepsis ${ }^{8,113}$. Interestingly, restoring IL-7 production by osteoblast stimulation ameliorated immune defects in mice subjected to CLP, as osteoblast ablation during sepsis is believed to cause lymphopaenia (owing to a lack of IL-7 production and thus decreased generation of lymphoid progenitors) ${ }^{210}$.

In ex vivo experiments using cells from patients with sepsis, rhIL-7 treatment substantially increased T lymphocyte functions (that is, an increase in proliferation, IFN $\gamma$ production and STAT5 phosphorylation and induction of BCL-2 protein production $)^{211}$. In a similar approach, rhIL-7 has been demonstrated to restore immunometabolic failure in lymphocytes from patients with sepsis by ameliorating changes in mTOR activation, whereas downstream TCR signalling was maintained ${ }^{49}$. In addition, IL-7 treatment also had positive effects in the treatment of cancer by enhancing the immune response in combination with anti-PD1 antibodies ${ }^{212}$. This result suggests that combining immunostimulatory therapies in patients with sepsis represents an effective therapeutic option ${ }^{209,210}$, as illustrated in a clinical case of mucormycosis ${ }^{199,213,214}$.

On the basis of these convincing preclinical results, a phase II multicentre randomized controlled trial assessing rhIL-7 treatment in patients with septic shock was designed and was conducted in parallel in the USA ${ }^{215}$ and in France ${ }^{216}$. The primary aim of this study was to confirm that rhIL-7 is safe and is able to increase the absolute lymphocyte count in immunosuppressed patients with sepsis. This clinical trial has ended, and the results are expected to be published in 2018 .

Immune checkpoint inhibitors. The PD1 receptor system constitutes a potent immunoregulatory pathway that negatively controls immune responses. This system comprises PD1 and its two ligands, PDL1 and PDL2. Immune checkpoint inhibitors are antibodies that target this key signalling pathway, and they are revolutionizing the treatment of cancer and, potentially, the treatment of chronic infectious diseases ${ }^{186,214}$. In patients with sepsis, several observational studies described an association between increased expression of PD1-related molecules in circulating immune cells and immune dysfunctions (such as lymphocyte proliferation) and deleterious outcomes (such as nosocomial infections or mortality). In addition, ex vivo studies in cells from patients with sepsis showed that PD1 or PDL1 pathway blockade decreased sepsisinduced immune dysfunctions ${ }^{108,217}$. In mice with sepsis, administration of anti-PD1 or anti-PDL1 antibodies increased bacterial clearance, reduced organ dysfunction, restored protective immune responses and improved survival $^{110,218-220}$. That said, the efficacy of anti-PD1 antibodies versus anti-PDL1 antibodies remains to be clearly delineated. To date, no randomized controlled trials evaluating immune checkpoint inhibitors in patients with sepsis have been published. A single case report described the use of a single dose of nivolumab in combination with repeated IFN $\gamma$ treatment in a 30-year old woman with invasive mucormycosis that was unresponsive to conventional therapy and who presented with immunosuppression ${ }^{199}$. Following treatment with this combination therapy, the patient improved slowly, did not show persistent signs of immunosuppression or residual infection and was finally discharged from the ICU on day 80 . Two clinical safety studies (phase Ib and phase IIa) with either nivolumab ${ }^{221}$ or BMS-936559 (an anti-PDL1 monoclonal antibody) ${ }^{222}$ have been completed in the treatment of patients with sepsis or septic shock, but have not yet been published. Considering these preliminary results and the large amounts of data obtained from cancer treatment regarding the safety and toxicity profile of anti-PD1 monoclonal antibodies, a large, international, multicentre randomized controlled trial of nivolumab is planned to start soon.

\section{Biomarkers and immunostimulation}

The international expert consensus is that the heterogeneity of patients with sepsis might be in part responsible for the failure of clinical trials of treatments for patients with sepsis ${ }^{223,224}$. This heterogeneity includes not only characteristics of the infection (type and virulence of the microorganism and the site of infection) and patient-specific parameters (such as age, comorbidities and genetic background) but also the immune status of each patient at a given moment, especially considering the rapidly evolving immune or inflammatory response after sepsis, which involves switching from a robust inflammatory response to profound immunosuppression. The need for biomarker stratification of patients thus seems a prerequisite for clinical trials to evaluate immunostimulation therapies in patients with sepsis, as it is unwise to further increase the immune response in patients who continue to suffer from a hyperinflammatory response ${ }^{223,224}$ (extensively reviewed elsewhere $\left.{ }^{86,87,225}\right)$. To date, absolute lymphocyte count and decreased expression of HLA-DR by monocytes seem to be the most robust markers for patient stratification in multicentre clinical trials. Both measurements are standardized, and their variations are associated with altered functions and deleterious outcomes (either nosocomial infection occurrence or mortality). Of note, alternative methods have been developed to evaluate HLA-DR expression owing to pre-analytical requirements and because flow cytometers are usually not available at all times in hospitals. For example, quantitative reverse transcription PCR (qRT-PCR) evaluation of the expression of major histocompatibility complex (MHC) class II-related genes represents a promising approach ${ }^{226}$, especially considering the current development of automated qRT-PCR systems. Technical specifications, such as standardization 
of results (interlaboratory assessment, use of reference genes), analytical specifications (reproducibility and coefficients of variation) and validation in large multicentre cohorts of patients in the ICU, are now expected. Another alternative lies in recent fully automated flow cytometers that offer dedicated applications, such as quantification of absolute $\mathrm{CD} 4^{+} \mathrm{T}$ lymphocyte numbers or evaluation of CD64 (also known as FCGR1A) expression in neutrophils ${ }^{227}$, which may be extended to measurements of monocyte HLA-DR expression ${ }^{228}$. In addition, in light of findings discussed in this Review, monitoring of MDSCs (or immature neutrophils) and potential stratification markers for anti-PD1 antibody treatments (for example, soluble PDL1 is already used in the treatment of cancer) seem more than desirable.

Another important challenge is to improve the effectiveness of therapies. Functional testing seems to be the gold standard because it directly measures ex vivo the capacity of a cell population to respond to an immune challenge. This testing might indicate whether a given therapy is truly effective in restoring immune functions in patients. Nevertheless, until recently, this testing was not feasible on a routine basis in the clinical setting owing to a lack of standardization. That said, among immune functional assays, measurement of intracellular cytokine production by flow cytometry seems promising, especially as new whole blood protocols have been developed. These rapid and robust techniques, which are potentially usable for patients with sepsis in clinics, provide substantial information regarding TNF production in monocytes ${ }^{229}$ or IL- 2 and IFN $\gamma$ production by $\mathrm{CD} 4^{+}$ cells and CD8 ${ }^{+}$cells in patients ${ }^{230}$.

\section{Conclusions and perspectives}

Although the description of sepsis-induced immune dysfunctions has improved over the past 10 years, numerous questions remain, and some aspects deserve further investigation. For example, evaluation of the role of immature myeloid cells in sepsis-induced immunosuppression will probably provide crucial information about the pathophysiology of sepsis, as these cells could represent the missing link between immunosenescence and sepsis. In line with this approach, long-term monitoring of immune status in patients after hospital discharge needs to be assessed with appropriate biomarkers. A precise description of the similarities between cancer and sepsis-induced immune dysfunctions, including metabolic aspects, would be worthwhile, as this analysis could establish a field of immunotherapy in the treatment of sepsis-induced immunosuppression. Most importantly, the forthcoming results of randomized controlled trials evaluating IL-7, GM-CSF and anti-PD1 antibodies for the treatment of sepsis will be informative for the design of experiments to unravel the molecular mechanisms of sepsis-induced immunosuppression. Finally, increasing data show that immune alterations similar to those in sepsis also occur after other severe non-infectious injuries, such as trauma, burns or surgery, which result in increased susceptibility to nosocomial infections ${ }^{231}$. Immunomonitoring approaches developed in the treatment of patients with sepsis may thus help to identify patients in the ICU for whom pre-emptive actions could be taken to decrease the risk of secondary infections. This approach may provide a novel way to treat and prevent nosocomial infections in patients in the ICU.
1. Singer, M. et al. The Third International Consensus Definitions for Sepsis and Septic Shock (Sepsis-3). JAMA 315, 801-810 (2016).

2. Deutschman, C. S. \& Tracey, K. J. Sepsis: current dogma and new perspectives. Immunity 40, 463-475 (2014).

3. Hotchkiss, R. S. et al. Sepsis and septic shock. Nat. Rev. Dis. Primers 2, 16045 (2016).

4. Reinhart, K. et al. Recognizing sepsis as a global health priority - a WHO resolution. N. Engl. J. Med. 377, 414-417 (2017).

5. van der Poll, T., van de Veerdonk, F. L., Scicluna, B. P. \& Netea, M. G. The immunopathology of sepsis and potential therapeutic targets. Nat. Rev. Immunol. 17 407-420 (2017)

6. Zeni, F., Freeman, B. \& Natanson, C. Antiinflammatory therapies to treat sepsis and septic shock: a reassessment. Crit. Care Med. 25. 1095-1100 (1997).

7. Hotchkiss, R. S., Monneret, G. \& Payen, D Immunosuppression in sepsis: a novel understanding of the disorder and a new therapeutic approach. Lancet Infect. Dis. 13, 260-268 (2013).

8. Hotchkiss, R. S., Monneret, G. \& Payen, D Sepsis-induced immunosuppression: from cellular dysfunctions to immunotherapy. Nat. Rev. Immunol. 13, 862-874 (2013)

9. Meakins, J. L. et al. Delayed hypersensitivity: indicator of acquired failure of host defenses in sepsis and trauma. Ann. Surg. 186, 241-250 (1977)

10. Christou, N. V. et al. The delayed hypersensitivity response and host resistance in surgical patients. 20 years later. Ann. Surg. 222, 534-548 (1995).

11. Munoz, C. et al. Dysregulation of in vitro cytokine production by monocytes during sepsis. J. Clin. Invest. 88, 1747-1754 (1991)

12. Hotchkiss, R. S. et al. Apoptotic cell death in patients with sepsis, shock, and multiple organ dysfunction. Crit. Care Med. 27, 1230-1251 (1999).
13. Hotchkiss, R. S. et al. Rapid onset of intestinal epithelial and lymphocyte apoptotic cell death in patients with trauma and shock. Crit. Care Med. 28 3207-3217 (2000)

14. Bone, R. C., Grodzin, C. J. \& Balk, R. A. Sepsis: a new hypothesis for pathogenesis of the disease process. Chest 112, 235-243 (1997)

15. Docke, W. D. et al. Monocyte deactivation in septic patients: restoration by IFN- $\gamma$ treatment. Nat. Med. 3, 678-681 (1997)

16. Munford, R. S. \& Pugin, J. Normal responses to injury prevent systemic inflammation and can be mmunosuppressive. Am. J. Respir. Crit. Care Med. 163, 316-321 (2001).

17. Hotchkiss, R. S. \& Nicholson, D. W. Apoptosis and caspases regulate death and inflammation in sepsis. Nat. Rev. Immunol. 6, 813-822 (2006).

18. Hotchkiss, R. S. et al. Adoptive transfer of apoptotic splenocytes worsens survival, whereas adoptive transfer of necrotic splenocytes improves survival in sepsis. Proc. Natl Acad. Sci. USA 100, 6724-6729 (2003).

19. Ayala, A et al. Apoptosis in sepsis: mechanisms clinical impact and potential therapeutic targets. Curr. Pharm. Des. 14, 1853-1859 (2008)

20. Hotchkiss, R. S., Coopersmith, C. M. \& Karl, I. E. Prevention of lymphocyte apoptosis - a potential treatment of sepsis? Clin. Infect. Dis. 41 (Suppl. 7) S465-S469 (2005)

21. Jorgensen, I., Rayamajhi, M. \& Miao, E. A. Programmed cell death as a defence against infection. Nat. Rev. Immunol. 17, 151-164 (2017).

22. Cadwell, K. Crosstalk between autophagy and inflammatory signalling pathways: balancing defence and homeostasis. Nat. Rev. Immunol. 16, 661-675 (2016).

23. Hsieh, Y. C., Athar, M. \& Chaudry, I. H. When apoptosis meets autophagy: deciding cell fate after trauma and sepsis. Trends Mol. Med. 15, 129-138 (2009).
24. Lin, C. W. et al. T-Cell autophagy deficiency increases mortality and suppresses immune responses after sepsis. PLoS ONE 9, e102066 (2014).

25. Oami, T. et al. Suppression of T cell autophagy results in decreased viability and function of T cells through accelerated apoptosis in a murine sepsis model. Crit. Care Med. 45, e77-e85 (2017).

26. Seeley, J.J. \& Ghosh, S. Molecular mechanisms of innate memory and tolerance to LPS. J. Leukoc. Biol. 101, 107-119 (2017)

27. Biswas, S. K. \& Lopez-Collazo, E. Endotoxin tolerance new mechanisms, molecules and clinical significance. Trends Immunol. 30, 475-487 (2009).

28. Wolk, K., Docke, W. D., von Baehr, V., Volk, H. D. \& Sabat, R. Impaired antigen presentation by human monocytes during endotoxin tolerance. Blood 96 218-223 (2000)

29. Shalova, I. N. et al. Human monocytes undergo functional re-programming during sepsis mediated by hypoxia-inducible factor- $1 \alpha$. Immunity 42, 484-498 (2015).

30. Allantaz-Frager, F. et al. Identification of biomarkers of response to IFNg during endotoxin tolerance: application to septic shock. PLOS ONE 8, e68218 (2013).

31. Morris, M. C., Gilliam, E. A. \& Li, L. Innate immune programing by endotoxin and its pathological consequences. Front. Immunol. 5, 680 (2015)

32. van 't Veer, C. et al. Induction of IRAK-M is associated with lipopolysaccharide tolerance in a human endotoxemia model. J. Immunol. 179, 7110-7120 (2007).

33. Sfeir, T., Saha, D. C., Astiz, M. \& Rackow, E. C. Role of interleukin-10 in monocyte hyporesponsiveness associated with septic shock. Crit. Care Med. 29 129-133 (2001)

34. Novakovic, B. et al. $\beta$-Glucan reverses the epigenetic state of LPS-induced immunological tolerance. Cell 167, 1354-1368.e14 (2016). 
35. Muller, M. M. et al. Global analysis of glycoproteins identifies markers of endotoxin tolerant monocytes and GPR84 as a modulator of TNF $\alpha$ expression. Sci. Rep. 7, 838 (2017).

36. Hoogendijk, A. J. et al. Sepsis patients display a reduced capacity to activate nuclear factor- $\mathrm{kB}$ in multiple cell types. Crit. Care Med. 45, e524-e531 (2017).

37. Netea, M. G. et al. Trained immunity: a program of innate immune memory in health and disease. Science 352, aaf1098 (2016)

38. Chavan, S. S. \& Tracey, K. J. Essential neuroscience in immunology. J. Immunol. 198, 3389-3397 (2017)

39. Sternberg, E. M. Neural regulation of innate immunity: a coordinated nonspecific host response to pathogens. Nat. Rev. Immunol. 6, 318-328 (2006).

40. Dalli, J., Colas, R. A., Arnardottir, H. \& Serhan, C. N. Vagal regulation of group 3 innate lymphoid cells and the immunoresolvent PCTR1 controls infection resolution. Immunity 46, 92-105 (2017).

41. Singer, B. H. et al. Cecal ligation and puncture results in long-term central nervous system myeloid inflammation. PLOS ONE 11, e0149136 (2016).

42. Martelli, D., Yao, S. T., McKinley, M. J. \& McAllen, R. M. Reflex control of inflammation by sympathetic nerves, not the vagus. J. Physiol. 592, 1677-1686 (2014).

43. Stolk, R. F. et al. Potentially inadvertent immunomodulation: norepinephrine use in sepsis. Am. J. Respir. Crit. Care Med. 194, 550-558 (2016)

44. Maclver, N. J., Michalek, R. D. \& Rathmell, J. C. Metabolic regulation of T lymphocytes. Annu. Rev. Immunol. 31, 259-283 (2013).

45. Stienstra, R., Netea-Maier, R. T., Riksen, N. P., Joosten, L. A. B. \& Netea, M. G. Specific and complex reprogramming of cellular metabolism in myeloid cells during innate immune responses. Cell Metab. 26 142-156 (2017)

46. Arts, R. J., Gresnigt, M. S., Joosten, L. A. \& Netea, M. G. Cellular metabolism of myeloid cells in sepsis. J. Leukoc. Biol. 101, 151-164 (2017)

47. Cheng, S. C. et al. mTOR- and HIF- $1 \alpha$-mediated aerobic glycolysis as metabolic basis for trained immunity. Science 345, 1250684 (2014).

48. Cheng, S. C. et al. Broad defects in the energy metabolism of leukocytes underlie immunoparalysis in sepsis. Nat. Immunol. 17, 406-413 (2016).

49. Venet, F. et al. IL-7 restores T lymphocyte immunometabolic failure in septic shock patients through mTOR activation. J. Immunol. 199 1606-1615 (2017).

50. Edwards, M. R. et al. Metabolic dysfunction in lymphocytes promotes postoperative morbidity. Clin. Sci. 129, 423-437 (2015).

51. Singer, M., De Santis, V., Vitale, D. \& Jeffcoate, W. Multiorgan failure is an adaptive, endocrine-mediated, metabolic response to overwhelming systemic inflammation. Lancet 364, 545-548 (2004).

52. Cazalis, M. A. et al. Early and dynamic changes in gene expression in septic shock patients: a genomewide approach. Intensive Care Med. Exp. 2, 20 (2014).

53. Xiao, W. et al. A genomic storm in critically injured humans. J. Exp. Med. 208, 2581-2590 (2011).

54. Davenport, E. E. et al. Genomic landscape of the individual host response and outcomes in sepsis: aprospective cohort study. Lancet Respir. Med. 4 259-271 (2016)

55. Carson, W. F., Cavassani, K. A., Dou, Y. \& Kunkel, S. L. Epigenetic regulation of immune cell functions during post-septic immunosuppression. Epigenetics 6 , 273-283 (2011)

56. Leentjens, J., Kox, M., van der Hoeven, J. G., Netea, M. G. \& Pickkers, P. Immunotherapy for the adjunctive treatment of sepsis: from immunosuppression to immunostimulation. Time for a paradigm change? Am. J. Respir. Crit. Care Med. 187, 1287-1293 (2013).

57. El Gazzar, M. et al. Chromatin-specific remodeling by HMGB 1 and linker histone $\mathrm{H} 1$ silences proinflammatory genes during endotoxin tolerance. Mol. Cell. Biol. 29, 1959-1971 (2009).

58. El Gazzar, M., Liu, T., Yoza, B. K. \& McCall, C. E. Dynamic and selective nucleosome repositioning during endotoxin tolerance. J. Biol. Chem. 285, 1259-1271 (2010).

59. Wen, H., Dou, Y., Hogaboam, C. M. \& Kunkel, S. L. Epigenetic regulation of dendritic cell-derived interleukin- 12 facilitates immunosuppression after a severe innate immune response. Blood 111, 1797-1804 (2008).
60. Carson, W. F.t. et al. Impaired CD4+ T-cell proliferation and effector function correlates with repressive histone methylation events in a mouse model of severe sepsis. Eur. J. Immunol. 40, 998-1010 (2010).

61. Cavassani, K. A. et al. The post sepsis-induced expansion and enhanced function of regulatory $\mathrm{T}$ cells create an environment to potentiate tumor growth. Blood 115, 4403-4411 (2010).

62. El Gazzar, M. \& McCall, C. E. MicroRNAs distinguish translational from transcriptional silencing during endotoxin tolerance. J. Biol. Chem. 285 20940-20951 (2010)

63. Vachharajani, V. T. et al. Sirtuins link inflammation and metabolism. J. Immunol. Res. 2016, 8167273 (2016).

64. Liu, T. F., Yoza, B. K., El Gazzar, M., Vachharajani, V. T. \& McCall, C. E. NAD ${ }^{+}$-dependent SIRT 1 deacetylase participates in epigenetic reprogramming during endotoxin tolerance. J. Biol. Chem. 286, 9856-9864 (2011).

65. Opal, S. M. et al. Pharmacological Sirt1 activation improves mortality and markedly alters transcriptiona profiles that accompany experimental sepsis. Shock 45, 411-418 (2016)

66. Guerin, E. et al. Circulating immature granulocytes with T-cell killing functions predict sepsis deterioration*. Crit. Care Med. 42, 2007-2018 (2014).

67. Pillay, J. et al. A subset of neutrophils in human systemic inflammation inhibits $\mathrm{T}$ cell responses through Mac-1. J. Clin. Invest. 122, 327-336 (2012)

68. Demaret, J. et al. Marked alterations of neutrophil functions during sepsis-induced immunosuppression. J. Leukoc. Biol. 98, 1081-1090 (2015).

69. Hampson, P. et al. Neutrophil dysfunction, immature granulocytes, and cell-free DNA are early biomarkers of sepsis in burn-injured patients: a prospective observational cohort study. Ann. Surg. 265 1241-1249 (2016)

70. Groeneveld, K. M. et al. Early decreased neutrophil responsiveness is related to late onset sepsis in multitrauma patients: an international cohort study. PLOS ONE 12, e0180145 (2017).

71. Gregoire, M. et al. Frontline Science: HMGB1 induces neutrophil dysfunction in experimental sepsis and in patients who survive septic shock. J. Leukoc. Biol. 101, 1281-1287 (2017)

72. Huang, X. et al. Identification of B7-H1 as a novel mediator of the innate immune/proinflammatory response as well as a possible myeloid cell prognostic biomarker in sepsis. J. Immunol. 192, 1091-1099 (2014).

73. Gabrilovich, D. I. Myeloid-derived suppressor cells Cancer Immunol. Res. 5, 3-8 (2017).

74. Ost, M. et al. Myeloid-derived suppressor cells in bacterial infections. Front. Cell. Infect. Microbiol. 6, 37 (2016).

75. Delano, M. J. et al. MyD88-dependent expansion of an immature GR-1 $+\mathrm{CD} 11 \mathrm{~b}^{+}$population induces T cell suppression and Th2 polarization in sepsis. J. Exp. Med. 204, 1463-1474 (2007).

76. Brudecki, L., Ferguson, D. A., McCall, C. E. \& El Gazzar, M. Myeloid-derived suppressor cells evolve during sepsis and can enhance or attenuate the systemic inflammatory response. Infect. Immun. 80, 2026-2034 (2012)

77. Albertsmeier, M. et al. Monocyte-dependent suppression of T-cell function in postoperative patients and abdominal sepsis. Shock 48, 651-656 (2017).

78. McPeak, M. B. et al. Myeloid cell-specific knockout of NFI-A improves sepsis survival. Infect. Immun. 85 e00066-17 (2017).

79. McPeak, M. B et al. Frontline Science: Myeloid cellspecific deletion of Cebpb decreases sepsis-induced immunosuppression in mice. J. Leukoc. Biol. 102 , 191-200 (2017).

80. Landoni, V. I. et al. Immature myeloid Gr-1 + CD $11 b^{+}$ cells from lipopolysaccharide-immunosuppressed mice acquire inhibitory activity in the bone marrow and migrate to lymph nodes to exert their suppressive function. Clin. Sci. 130, 259-271 (2016).

81. Llitjos, J. F. et al. Sepsis-induced expansion of granulocytic myeloid-derived suppressor cells promotes tumour growth through Toll-like receptor 4 J. Pathol. 239, 473-483 (2016).

82. Mathias, B. et al. Human myeloid-derived suppressor cells are associated with chronic immune suppression after severe sepsis/septic shock. Ann. Surg. 265 827-834 (2016)
83. Janols, H. et al. A high frequency of MDSCs in sepsis patients, with the granulocytic subtype dominating in gram-positive cases. J. Leukoc. Biol. 96, 685-693 (2014).

84. Uhel, F. et al. Early expansion of circulating granulocytic myeloid-derived suppressor cells predicts development of nosocomial infections in septic patients. Am. J. Respir. Crit. Care Med. 196, 315-327 (2017).

85. Dumitru, C. A., Moses, K., Trellakis, S., Lang, S. \& Brandau, S. Neutrophils and granulocytic myeloidderived suppressor cells: immunophenotyping, cell biology and clinical relevance in human oncology. Cancer Immunol. Immunother. 61, 1155-1167 (2012).

86. Monneret, G. \& Venet, F. Sepsis-induced immune alterations monitoring by flow cytometry as a promising tool for individualized therapy. Cytom. $B$ Clin. Cytom 90, 376-386 (2015).

87. Venet, F., Lukaszewicz, A. C., Payen, D., Hotchkiss, R. \& Monneret, G. Monitoring the immune response in sepsis: a rational approach to administration of immunoadjuvant therapies. Curr. Opin. Immunol. 25, 477-483 (2013).

88. Galbraith, N., Walker, S., Carter, J. \& Polk, H. C. Jr. Past, present, and future of augmentation of monocyte function in the surgical patient. Surg. Infect. 17, 563-569 (2016)

89. Guignant, C. et al. Programmed death-1 levels correlate with increased mortality, nosocomial infection and immune dysfunctions in septic shock patients. Crit. Care 15, R99 (2011).

90. Zhang, Y. et al. Upregulation of programmed death-1 on T cells and programmed death ligand- 1 on monocytes in septic shock patients. Crit. Care 15, R70 (2011)

91. Shao, R. et al. Monocyte programmed death ligand-1 expression after 3-4 days of sepsis is associated with risk stratification and mortality in septic patients: a prospective cohort study. Crit. Care 20, 124 (2016).

92. Bernsmeier, C. et al. CD 14+ ${ }^{+}$CD 15-HLA-DR - myeloidderived suppressor cells impair antimicrobial responses in patients with acute-on-chronic liver failure. Gut http://dx.doi.org/10.1136 gutjnl-2017-314184 (2017).

93. Roquilly, A. \& Villadangos, J. A. The role of dendritic cell alterations in susceptibility to hospital-acquired infections during critical-illness related immunosuppression. Mol. Immunol. 68, 120-123 (2015).

94. Guisset, O. et al. Decrease in circulating dendritic cells predicts fatal outcome in septic shock. Intensive Care Med. 33, 148-152 (2007).

95. Grimaldi, D. et al. Profound and persistent decrease of circulating dendritic cells is associated with ICU acquired infection in patients with septic shock Intensive Care Med. 37, 1438-1446 (2011)

96. Wen, H., Schaller, M. A., Dou, Y., Hogaboam, C. M. \& Kunkel, S. L. Dendritic cells at the interface of innate and acquired immunity: the role for epigenetic changes. J. Leukoc. Biol. 83, 439-446 (2008)

97. Strother, R. K. et al. Polymicrobial sepsis diminishes dendritic cell numbers and function directly contributing to impaired primary CD8 T cell responses In Vivo. J. Immunol. 197, 4301-4311 (2016).

98. Meng, Y. et al. CD155 blockade improves survival in experimental sepsis by reversing dendritic cell dysfunction. Biochem. Biophys. Res. Commun. 490 283-289 (2017)

99. Huang, X., Venet, F., Chung, C. S., Lomas-Neira, J. $\&$ Ayala, A. Changes in dendritic cell function in the immune response to sepsis. Cell- and tissue-based therapy. Expert Opin. Biol. Ther. 7, 929-938 (2007).

100. Roquilly, A. et al. Local modulation of antigenpresenting cell development after resolution of pneumonia induces long-term susceptibility to secondary infections. Immunity 47, 135-147.e5 (2017).

101. Venet, F. et al. Early assessment of leukocyte alterations at diagnosis of septic shock. Shock 34 358-363 (2010)

102. Le Tulzo, Y. et al. Early circulating lymphocyte apoptosis in human septic shock is associated with poor outcome. Shock 18, 487-494 (2002).

103. Drewry, A. M. et al. Persistent lymphopenia after diagnosis of sepsis predicts mortality. Shock $\mathbf{4 2}$, 383-391 (2014).

104. Chung, K. P. et al. Severe lymphopenia is associated with elevated plasma interleukin-15 levels and increased mortality during severe sepsis. Shock 43 569-575 (2015). 
105. Adrie, C. et al. Persistent lymphopenia is a risk factor for ICU-acquired infections and for death in ICV patients with sustained hypotension at admission. Ann. Intensive Care 7, 30 (2017).

106. Souza-Fonseca-Guimaraes, F., Parlato, M., Fitting, C., Cavaillon, J. M. \& Adib-Conquy, M. NK cell tolerance to TLR agonists mediated by regulatory T cells after polymicrobial sepsis. J. Immunol. 188, 5850-5858 (2012).

107. Deknuydt, F., Roquilly, A., Cinotti, R., Altare, F. \& Asehnoune, K. An in vitro model of mycobacterial granuloma to investigate the immune response in brain-injured patients. Crit. Care Med. 41, 245-254 (2013).

108. Patera, A. C. et al. Frontline Science: Defects in immune function in patients with sepsis are associated with PD-1 or PD-L1 expression and can be restored by antibodies targeting PD-1 or PD-L1. J. Leukoc. Biol. 100, 1239-1254 (2016)

109. Hou, H. et al. Tim-3 negatively mediates natural killer cell function in LPS-induced endotoxic shock. PLOS ONE 9, e110585 (2014).

110. Shindo, Y. et al. Anti-PD-L1 peptide improves survival in sepsis. J. Surg. Res. 208, 33-39 (2017).

111. Chiche, L. et al. Interferon-gamma production by natural killer cells and cytomegalovirus in critically ill patients. Crit. Care Med. 40, 3162-3169 (2012).

112. Wong, C. H., Jenne, C. N., Lee, W. Y., Leger, C. \& Kubes, P. Functional innervation of hepatic iNKT cells is immunosuppressive following stroke. Science 334, 101-105 (2011)

113. Shindo, Y. et al. Interleukin 7 immunotherapy improves host immunity and survival in a two-hit model of Pseudomonas aeruginosa pneumonia. J. Leukoc. Biol. 101, 543-554 (2017).

114. Grimaldi, D. et al. Specific MAIT cell behaviour among innate-like T lymphocytes in critically ill patients with severe infections. Intensive Care Med. 40, 192-201 (2014).

115. Mohr, A. et al. Sepsis leads to a reduced antigenspecific primary antibody response. Eur. J. Immunol. 42, 341-352 (2012).

116. Kelly-Scumpia, K. M. et al. B cells enhance early innate immune responses during bacterial sepsis. J. Exp. Med. 208, 1673-1682 (2011).

117. Rauch, P. J. et al. Innate response activator B cells protect against microbial sepsis. Science 335 , 597-601 (2012)

118. Weber, G. F. et al. Interleukin-3 amplifies acute inflammation and is a potential therapeutic target in sepsis. Science 347, 1260-1265 (2015).

119. Aziz, M., Holodick, N. E., Rothstein, T. L. \& Wang, P. B-1 a cells protect mice from sepsis: critical role of CREB. J. Immunol. 199, 750-760 (2017).

120. Mauri, C. \& Menon, M. Human regulatory B cells in health and disease: therapeutic potential. J. Clin. Invest. 127, 772-779 (2017).

121. Shankar-Hari, M. et al. Activation-associated accelerated apoptosis of memory B cells in critically il patients with sepsis. Crit. Care Med. 45, 875-882 (2017).

122. Suzuki, K. et al. Reduced immunocompetent B cells and increased secondary infection in elderly patients with severe sepsis. Shock 46, 270-278 (2016).

123. Giamarellos-Bourboulis, E. J. et al. Kinetics of circulating immunoglobulin $\mathrm{M}$ in sepsis: relationship with final outcome. Crit. Care 17, R247 (2013).

124. Pan, X., Ji, Z. \& Xue, J. Percentage of peripheral $\mathrm{CD} 19+\mathrm{CD} 24^{\mathrm{hi}} \mathrm{CD} 38^{\text {hi }}$ regulatory $\mathrm{B}$ cells in neonatal sepsis patients and its functional implication. Med. Sci. Monit. 22, 2374-2378 (2016).

125. Cabrera-Perez, J., Condotta, S. A., Badovinac, V. P. $\&$ Griffith, T. S. Impact of sepsis on CD4 T cell immunity. J. Leukoc. Biol. 96, 767-777 (2014).

126. Danahy, D. B., Strother, R. K., Badovinac, V. P. \& Griffith, T. S. Clinical and experimental sepsis impairs CD8 T-cell-mediated immunity. Crit. Rev. Immunol. 36 57-74 (2016)

127. Boomer, J. S. et al. Immunosuppression in patients who die of sepsis and multiple organ failure. JAMA 306, 2594-2605 (2011).

128. Spec, A. et al. T cells from patients with Candida sepsis display a suppressive immunophenotype. Crit. Care 20, 15 (2016).

129. Demaret, J. et al. STAT5 phosphorylation in T cell subsets from septic patients in response to recombinant human interleukin-7: a pilot study. J. Leukoc. Biol. 97, 791-796 (2015)

130. van der Heide, V., Mohnle, P., Rink, J., Briegel, J. \& Kreth, S. Down-regulation of microRNA-31 in CD4 $\mathrm{T}$ cells contributes to immunosuppression in human sepsis by promoting $\mathrm{T}_{H} 2$ skewing. Anesthesiology 124, 908-922 (2016)

131. Boomer, J. S., Shuherk-Shaffer, J., Hotchkiss, R. S \& Green, J. M. A prospective analysis of lymphocyte phenotype and function over the course of acute sepsis. Crit. Care 16, R112 (2012).

132. Venet, F. et al. Decreased T cell repertoire diversity in sepsis: a preliminary study. Crit. Care Med. 41, 111-119 (2013)

133. Venet, F. et al. Increased circulating regulatory $T$ cells $\left(\mathrm{CD} 4{ }^{+} \mathrm{CD} 25^{+} \mathrm{CD} 127-\right)$ contribute to lymphocyte anergy in septic shock patients. Intensive Care Med. 35, 678-686 (2009).

134. Venet, F. et al. Regulatory T cell populations in sepsis and trauma. J. Leukoc. Biol. 83, 523-535 (2008).

135. Nascimento, D. C. et al. Role of regulatory T cells in long-term immune dysfunction associated with sever sepsis. Crit. Care Med. 38, 1718-1725 (2010).

136. Huang, H. et al. High circulating CD39+ regulatory $T$ cells predict poor survival for sepsis patients. Int. J. Infect. Dis. 30, 57-63 (2014).

137. Nascimento, D. C. et al. IL-33 contributes to sepsisinduced long-term immunosuppression by expanding the regulatory T cell population. Nat. Commun. 8, 14919 (2017).

138. Hotchkiss, R. S. et al. Sepsis-induced apoptosis causes progressive profound depletion of $\mathrm{B}$ and $\mathrm{CD} 4^{+} \mathrm{T}$ lymphocytes in humans. J. Immunol. 166, 6952-6963 (2001).

139. Felmet, K. A., Hall, M. W., Clark, R. S., Jaffe, R. \& Carcillo, J. A. Prolonged lymphopenia, lymphoid depletion, and hypoprolactinemia in children with nosocomial sepsis and multiple organ failure. J. Immunol. 174, 3765-3772 (2005)

140. Muehlstedt, S. G., Lyte, M. \& Rodriguez, J. L. Increased IL-10 production and HLA-DR suppression in the lungs of injured patients precede the development of nosocomial pneumonia. Shock 17 443-450 (2002).

141. Chalk, K. et al. Dysfunction of alveolar macrophages after cardiac surgery and postoperative pneumonia? - An observational study. Crit. Care 17, R285 (2013).

142. Faivre, V., Lukaszewicz, A. C. \& Payen, D. Downregulation of blood monocyte HLA-DR in ICU patients is also present in bone marrow cells. PLOS ONE 11, e0164489 (2016).

143. Skirecki, T. et al. Early severe impairment of hematopoietic stem and progenitor cells from the bone marrow caused by CLP sepsis and endotoxemia in a humanized mice model. Stem Cell Res. Ther. 6 142 (2015)

144. Rodriguez, S. et al. Dysfunctional expansion of hematopoietic stem cells and block of myeloid differentiation in lethal sepsis. Blood 114 4064-4076 (2009)

145. Netzer, C. et al. Apoptotic diminution of immature single and double positive thymocyte subpopulations contributes to thymus involution during murine polymicrobial sepsis. Shock 48, 215-226 (2017).

146. Kong, Y. et al. Sepsis-induced thymic atrophy is associated with defects in early lymphopoiesis. Stem Cells 34, 2902-2915 (2016).

147. Gruver, A. L., Ventevogel, M. S. ¿ Sempowski, G. D. Leptin receptor is expressed in thymus medulla and leptin protects against thymic remodeling during endotoxemia-induced thymus involution. J. Endocrinol. 203, 75-85 (2009)

148. Torgersen, C. et al. Macroscopic postmortem findings in 235 surgical intensive care patients with sepsis. Anesth. Analg 108, 1841-1847 (2009).

149. Landelle, C. et al. Nosocomial infection after septic shock among intensive care unit patients. Infect. Control Hosp. Epidemiol. 29, 1054-1065 (2008)

150. van Vught, L. A. et al. Incidence, risk factors, and attributable mortality of secondary infections in the intensive care unit after admission for sepsis. JAMA 315, 1469-1479 (2016).

151. Koch, R. M. et al. Patterns in bacterial- and viralinduced immunosuppression and secondary infections in the ICU. Shock 47, 5-12 (2017).

152. Monneret, G., Venet, F., Kullberg, B. J. \& Netea, M. G ICU-acquired immunosuppression and the risk for secondary fungal infections. Med. Mycol. 49 (Suppl. 1), S17-S23 (2011)

153. Segal, B. H. Aspergillosis. N. Engl. J. Med. 360 , 1870-1884 (2009).

154. Walton, A. H. et al. Reactivation of multiple viruses in patients with sepsis. PLOS ONE 9, e98819 (2014).
155. Ong, D. S. Y. et al. Epidemiology of multiple herpes viremia in previously immunocompetent patients with septic shock. Clin. Infect. Dis. 64, 1204-1210 (2017)

156. Luyt, C. E., Combes, A., Nieszkowska, A., Trouillet, J. L. $\&$ Chastre, J. Viral infections in the ICU. Curr. Opin. Crit. Care 14, 605-608 (2008).

157. Ong, D. S. et al. Cytomegalovirus reactivation and mortality in patients with acute respiratory distress syndrome. Intensive Care Med. 42, 333-341 (2016)

158. Kalil, A. C. \& Florescu, D. F. Prevalence and mortality associated with cytomegalovirus infection in nonimmunosuppressed patients in the intensive care unit. Crit. Care Med. 37, 2350-2358 (2009).

159. Chiche, L. et al. Active cytomegalovirus infection is common in mechanically ventilated medical intensive care unit patients. Crit. Care Med. 37, 1850-1857 (2009).

160. Ong, D. S. et al. Cytomegalovirus seroprevalence as a risk factor for poor outcome in acute respiratory distress syndrome*. Crit. Care Med. 43, 394-400 (2015).

161. Kalil, A. C., Sun, J. \& Florescu, D. F. The importance of detecting cytomegalovirus infections in studies evaluating new therapies for severe sepsis. Crit. Care Med. 38, S663-667 (2010).

162. Goodwin, A. J., Rice, D. A Simpson, K. N. \& Ford, D. W. Frequency, cost, and risk factors of readmissions among severe sepsis survivors. Crit. Care Med. 43, 738-746 (2015).

163. Prescott, H. C., Langa, K. M., Liu, V., Escobar, G. J. \& Iwashyna, T. J. Increased 1-year healthcare use in survivors of severe sepsis. Am. J. Respir. Crit. Care Med. 190, 62-69 (2014).

164. Shankar-Hari, M. \& Rubenfeld, G. D. Understanding long-term outcomes following sepsis: implications and challenges. Curr. Infect. Dis. Rep. 18, 37 (2016).

165. Chang, D. W., Tseng, C. H. \& Shapiro, M. F. Rehospitalizations following sepsis: common and costly. Crit. Care Med. 43, 2085-2093 (2015).

166. Ortego, A. et al. Hospital-based acute care use in survivors of septic shock. Crit. Care Med. 43 729-737 (2015).

167. Prescott, H. C., Osterholzer, J. J., Langa, K. M. Angus, D. C. \& Iwashyna, T. J. Late mortality after sepsis: propensity matched cohort study. BMJ 353 . i2375 (2016).

168. Sun, A. et al. Association between index hospitalization and hospital readmission in sepsis survivors. Crit Care Med. 44, 478-487 (2016).

169. Wang, H. E. et al. Long-term mortality after community-acquired sepsis: a longitudinal populationbased cohort study. BMJ Open 4, e004283 (2014).

170. Linder, A. et al. Long-term (10-year) mortality of younger previously healthy patients with severe sepsis/septic shock is worse than that of patients with nonseptic critical illness and of the general population. Crit. Care Med. 42, 2211-2218 (2014).

171. Mira, J. C. et al. Sepsis pathophysiology, chronic critical illness, and persistent inflammationimmunosuppression and catabolism syndrome. Crit. Care Med. 45, 253-262 (2017).

172. Delano, M. J. \& Ward, P. A. Sepsis-induced immune dysfunction: can immune therapies reduce mortality? J. Clin. Invest. 126, 23-31 (2016).

173. Nomellini, V., Kaplan, L. J., Sims, C. A. \& Caldwell, C. C. Chronic critical illness and persistent inflammation: what can we learn from the elderly, injured, septic, and malnourished? Shock http://dx. doi.org/10.1097/SHK.0000000000000939 (2017).

174. Zorio, V. et al. Assessment of sepsis-induced immunosuppression at ICU discharge and 6 months after ICU discharge. Ann. Intensive Care 7, 80 (2017).

175. Yende, S. \& Angus, D. C. Long-term outcomes from sepsis. Curr. Infect. Dis. Rep. 9, 382-386 (2007).

176. Arens, C. et al. Sepsis-induced long-term immune paralysis - results of a descriptive, explorative study. Crit. Care 20, 93 (2016)

177. Borken, F. et al. Chronic critical illness from sepsis is associated with an enhanced TCR response. J. Immunol. 198, 4781-4791 (2017).

178. Oliveira, N. M. et al. Sepsis induces telomere shortening: a potential mechanism responsible for delayed pathophysiological events in sepsis survivors? Mol. Med. http://dx.doi.org/10.2119/ molmed. 2016.00225 (2016)

179. Nguyen, V., Mendelsohn, A. ¿ Larrick, J. W. Interleukin-7 and immunosenescence. J. Immunol. Res. 2017, 4807853 (2017)

180. Chaudhry, M. S., Velardi, E., Dudakov, J. A. \& van den Brink, M. R. Thymus: the next (re)generation Immunol. Rev. 271, 56-71 (2016). 
181. Shakoory, B. et al. Interleukin-1 receptor blockade is associated with reduced mortality in sepsis patients with features of macrophage activation syndrome: reanalysis of a prior phase III trial. Crit. Care Med. 44 , 275-281 (2016)

182. Panacek, E. A. et al. Efficacy and safety of the monoclonal anti-tumor necrosis factor antibody F(ab')2 fragment afelimomab in patients with severe sepsis and elevated interleukin-6 levels. Crit. Care Med. 32, 2173-2182 (2004)

183. Eichacker, P. Q. et al. Risk and the efficacy of antiinflammatory agents: retrospective and confirmatory studies of sepsis. Am. J. Respir. Crit. Care Med. 166, 1197-1205 (2002).

184. Benjamim, C. F., Lundy, S. K., Lukacs, N. W. Hogaboam, C. M. \& Kunkel, S. L. Reversal of longterm sepsis-induced immunosuppression by dendritic cells. Blood 105, 3588-3595 (2005).

185. Unsinger, J. et al. Interleukin-7 ameliorates immune dysfunction and improves survival in a 2-hit model of fungal sepsis. J. Infect. Dis. 206, 606-616 (2012).

186. Dyck, L. \& Mills, K. H. G. Immune checkpoints and their inhibition in cancer and infectious diseases. Eur. J. Immunol. 47, 765-779 (2017).

187. Hotchkiss, R. S. \& Moldawer, L. L. Parallels between cancer and infectious disease. N. Engl. J. Med. 371 , 380-383 (2014)

188. Shekarian, T. et al. Pattern recognition receptors: immune targets to enhance cancer immunotherapy. Ann. Oncol. 28, 1756-1766 (2017).

189. Roquilly, A. et al. TLR-4 agonist in post-haemorrhage pneumonia: role of dendritic and natural killer cells. Eur. Respir. J. 42, 1365-1378 (2013).

190. Davis, C. G. et al. TLR3 agonist improves survival to secondary pneumonia in a double injury model. J. Surg. Res. 182, 270-276 (2013).

191. Chen, G. H. et al. Intrapulmonary TNF gene therapy reverses sepsis-induced suppression of lung antibacterial host defense. J. Immunol. 165 6496-6503 (2000).

192. Wu, J. et al. The efficacy of thymosin alpha 1 for severe sepsis (ETASS): a multicenter, single-blind, randomized and controlled trial. Crit. Care 17, R8 (2013)

193. US National Library of Medicine. ClinicalTrials.gov http://www.clinicaltrials.gov/ct2/show/NCT02867267 (2017).

194. Hutchins, N. A., Unsinger, J., Hotchkiss, R. S. \& Ayala, A. The new normal: immunomodulatory agents against sepsis immune suppression. Trends Mol. Med 20, 224-233 (2014)

195. Nakos, G. et al. Immunoparalysis in patients with severe trauma and the effect of inhaled interferongamma. Crit. Care Med. 30, 1488-1494 (2002).

196. Nalos, M. et al. Immune effects of interferon gamma in persistent staphylococcal sepsis. Am. J. Respir. Crit. Care Med. 185, 110-112 (2012)

197. Lukaszewicz, A. C. et al. Monocytic HLA-DR expression in intensive care patients: interest for prognosis and secondary infection prediction. Crit. Care Med. 37 , 2746-2752 (2009).

198. Delsing, C. E. et al. Interferon-gamma as adjunctive immunotherapy for invasive fungal infections: a case series. BMC Infect. Dis. 14, 166 (2014).

199. Grimaldi, D., Pradier, O., Hotchkiss, R. S. \& Vincent, J. L. Nivolumab plus interferon-gamma in the treatment of intractable mucormycosis. Lancet Infect. Dis. 17, 18 (2017)
200. Cutino-Moguel, M. T., Eades, C., Rezvani, K. $\varnothing$ Armstrong-James, D. Immunotherapy for infectious diseases in haematological immunocompromise. Br. J. Haematol. 177, 348-356 (2017).

201. Hall, M. W. et al. Immunoparalysis and nosocomial infection in children with multiple organ dysfunction syndrome. Intensive Care Med. 37, 525-532 (2011).

202. Nelson, L. A. Use of granulocyte-macrophage colonystimulating factor to reverse anergy in otherwise immunologically healthy children. Ann. Allergy Asthma Immunol. 98, 373-382 (2007)

203. Drossou-Agakidou, V. et al. In vivo effect of rhGM-CSF And rhG-CSF on monocyte HLA-DR expression of septic neonates. Cytokine 18, 260-265 (2002).

204. Protti, A. et al. Granulocyte-macrophage colony stimulating factor for non-resolving legionellosis. Anaesth. Intensive Care 42, 804-806 (2014).

205. Meisel, C. et al. Granulocyte-macrophage colonystimulating factor to reverse sepsis-associated immunosuppression: a double-blind, randomized, placebo-controlled multicenter trial. Am. J. Respir Crit. Care Med. 180, 640-648 (2009).

206. Mathias, B., Szpila, B. E., Moore, F. A., Efron, P. A \& Moldawer, L. L. A. Review of GM-CSF therapy in sepsis. Medicine 94, e2044 (2015).

207. US National Library of Medicine. ClinicalTrials.gov http://clinicaltrials.gov/ct2/show/NCT02361528 (2016).

208. Mackall, C. L., Fry, T. J. \& Gress, R. E. Harnessing the biology of IL-7 for therapeutic application. Nat. Rev. Immunol. 11, 330-342 (2011).

209. Lundstrom, W., Fewkes, N. M. \& Mackall, C. L. IL-7 in human health and disease. Semin. Immunol. 24 218-224 (2012).

210. Terashima, A. et al. Sepsis-induced osteoblast ablation causes immunodeficiency. Immunity 44, 1434-1443 (2016).

211. Venet, F. et al. IL-7 restores lymphocyte functions in septic patients. J. Immunol. 189, 5073-5081 (2012)

212. Pauken, K. E. et al. Epigenetic stability of exhausted $T$ cells limits durability of reinvigoration by PD-1 blockade. Science 354, 1160-1165 (2016).

213. Shindo, Y., Unsinger, J., Burnham, C. A., Green, J. M. \& Hotchkiss, R. S. Interleukin-7 and anti-programmed cell death 1 antibody have differing effects to reverse sepsis-induced immunosuppression. Shock 43 , 334-343 (2015)

214. Yang, Y. Cancer immunotherapy: harnessing the immune system to battle cancer. J. Clin. Invest. 125, 3335-3337 (2015)

215. US National Library of Medicine. ClinicalTrials.gov http://www.clinicaltrials.gov/ct2/show/NCT02797431 (2017)

216. US National Library of Medicine. ClinicalTrials.gov http://www.clinicaltrials.gov/ct2/show/NCT02640807 (2017).

217. Chang, K. et al. Targeting the programmed cell death 1: programmed cell death ligand 1 pathway reverses $\mathrm{T}$ cell exhaustion in patients with sepsis. Crit. Care 18 R3 (2014).

218. Chang, K. C. et al. Blockade ofthe negative co-stimulatory molecules PD-1 and CTLA-4 improves survival in primary and secondary fungal sepsis. Crit. Care 17, R85 (2013).

219. Brahmamdam, P. et al. Delayed administration of anti-PD-1 antibody reverses immune dysfunction and improves survival during sepsis. J. Leukoc. Biol. 88 233-240 (2010)

220. Zhang, Y. et al. PD-L1 blockade improves survival in experimental sepsis by inhibiting lymphocyte apoptosis and reversing monocyte dysfunction. Crit. Care 14, R220 (2010).

221. US National Library of Medicine. ClinicalTrials.gov http://www.clinicaltrials.gov/ct2/show/NCT02960854 (2017).

222. US National Library of Medicine. ClinicalTrials.gov http://www.clinicaltrials.gov/ct2/show/NCT02576457 (2017).

223. Cohen, J. et al. Sepsis: a roadmap for furure research Lancet Infect. Dis. 15, 581-614 (2015).

224. Perner, A. et al. The intensive care medicine research agenda on septic shock. Intensive Care Med. $\mathbf{4 3}$ 1294-1305 (2017)

225. Monneret, G., Venet, F., Pachot, A. \& Lepape, A. Monitoring immune dysfunctions in the septic patient: a new skin for the old ceremony. Mol. Med. 14 64-78 (2008).

226. Peronnet, E. et al. Association between mRNA expression of CD74 and IL10 and risk of ICU-acquired infections: a multicenter cohort study. Intensive Care Med. 43, 1013-1020 (2017).

227. Sprung, C. L. et al. Comparison of cd64 levels performed by the facs and accellix systems. Intensive Care Med Exp 3 (Suppl. 1), A1012 (2015).

228. Zouiouich, M., Gossez, M., Venet, F., Rimmele, T. \& Monneret, G. Automated bedside flow cytometer for mHLA-DR expression measurement: a comparison study with reference protocol. Intensive Care Med. Exp. 5, 39 (2017).

229. Monneret, G. et al. Novel approach in monocyte intracellular TNF measurement: application to sepsisinduced immune alterations. Shock 47, 318-322 (2016).

230. Letessier, W. et al. Decreased intra-lymphocyte cytokines measurement in septic shock patients: a proof of concept study in whole blood. Cytokine http://dx.doi.org/10.1016/j.cyto.2017.09.029 (2017).

231. Rol, M. L. et al. The REAnimation Low Immune Status Markers (REALISM) project: a protocol for broad characterisation and follow-up of injuryinduced immunosuppression in intensive care unit (ICU) critically ill patients. BMJ Open 7, e015734 (2017).

\section{Acknowledgements}

Owing to space limitations, the authors cannot include an exhaustive discussion of studies on sepsis-induced immunosuppression. The authors sincerely apologize for works not cited in this manuscript.

\section{Author contributions}

Both authors researched data for the article, contributed sub stantially to discussion of the article's content, wrote the article and reviewed and/or edited the manuscript before submission

Competing interest statement

The authors declare no competing interests.

\section{Publisher's note}

Springer Nature remains neutral with regard to jurisdictional claims in published maps and institutional affiliations. 